\title{
Accelerated Complex-Step Finite Difference for Expedient Deformable Simulation
}

\author{
RAN LUO, The University of New Mexico \\ WEIWEI XU*, State Key Lab of CAD \& CG, Zhejiang University \\ TIANJIA SHAO, University of Leeds \\ HONGYI XU, Google \\ YIN YANG, The University of New Mexico
}

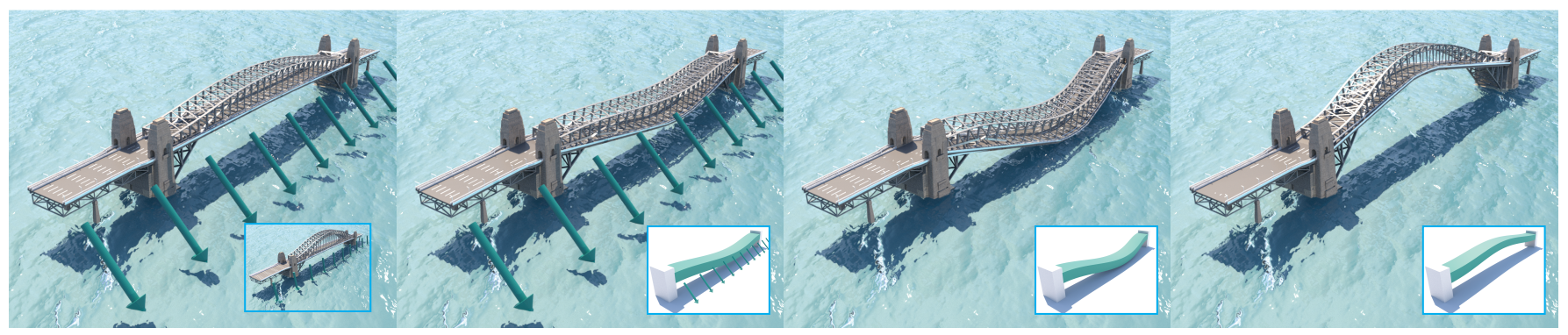

Fig. 1. We present an accelerated complex-step finite difference algorithm, which efficiently computes highly accurate numerical derivative. This method can be coupled with the Cauchy-Riemann formula to allow us to fully exploit existing (real-valued) linear algebra libraries to evaluate derivatives of tensor-valued functions. This figure reports an example of designing vibration frequency of a bridge model (21, 414 elements) by changing its geometry. The target frequency is visualized on a rectangular beam. Given an external force field, the bridge oscillates under the same frequency as the beam model does.

In deformable simulation, an important computing task is to calculate the gradient and derivative of the strain energy function in order to infer the corresponding internal force and tangent stiffness matrix. The standard numerical routine is the finite difference method, which evaluates the target function multiple times under a small real-valued perturbation. Unfortunately, the subtractive cancellation prevents us from setting this perturbation sufficiently small, and the regular finite difference is doomed for computing problems requiring a high-accuracy derivative evaluation. In this paper, we graft a new finite difference scheme, namely the complex-step finite difference (CSFD), with physics-based animation. CSFD is based on the complex Taylor series expansion, which avoids subtractions in first-order derivative approximation. As a result, one can use a very small perturbation to calculate the numerical derivative that is as accurate as its analytic counterpart. We accelerate the original CSFD method so that it is also as efficient as the analytic derivative. This is achieved by discarding high-order error terms, decoupling real and imaginary calculations, replacing costly functions based on the theory of equivalent infinitesimal, and isolating the propagation of the perturbation in composite/nesting functions. CSFD can be further augmented with multicomplex Taylor expansion and Cauchy-Riemann formula to handle higher-order derivatives and tensor-valued functions. We demonstrate the accuracy, convenience, and efficiency of this new numerical routine

${ }^{*}$ Corresponding author

Permission to make digital or hard copies of all or part of this work for personal or classroom use is granted without fee provided that copies are not made or distributed for profit or commercial advantage and that copies bear this notice and the full citation on the first page. Copyrights for components of this work owned by others than ACM must be honored. Abstracting with credit is permitted. To copy otherwise, or republish, to post on servers or to redistribute to lists, requires prior specific permission and/or a fee. Request permissions from permissions@acm.org.

(c) 2019 Association for Computing Machinery.

0730-0301/2019/11-ART160 \$15.00

https://doi.org/10.1145/3355089.3356493 in the context of deformable simulation - one can easily deploy a robust simulator for general hyperelastic materials, including user-crafted ones to cater specific needs in different applications. Higher-order derivatives of the energy can be readily computed to construct modal derivative bases for reduced real-time simulation. Inverse simulation problems can also be conveniently solved using gradient/Hessian-based optimization procedures.

CCS Concepts: • Computing methodologies $\rightarrow$ Physical simulation; - Mathematics of computing $\rightarrow$ Numerical analysis; Numerical differentiation;

Additional Key Words and Phrases: Physics-based simulation, Deformable model, Numerical differentiation, Finite difference

\section{ACM Reference format:}

Ran Luo, Weiwei Xu, Tianjia Shao, Hongyi Xu, and Yin Yang. 2019. Accelerated Complex-Step Finite Difference for Expedient Deformable Simulation. ACM Trans. Graph. 38, 6, Article 160 (November 2019), 16 pages. https://doi.org/10.1145/3355089.3356493

\section{INTRODUCTION}

As an essential computing task in physics-based simulation, the evaluation of various derivatives like total derivative, partial derivative, directional derivative, second- or high-order derivatives, etc. often stands out as a significant technical or implementation obstacle. Normally, people incline to infer an exact formula of derivative functions, which gives the best efficiency and accuracy. However, there are also many situations where a closed-form expression of the target function is not available, or deriving its actual derivative is too involved for just performing preliminary proof-of-concept trials. The numerical derivative is then preferred. 
The commonly used strategy for numerical derivative is the finite difference method. For instance, the forward difference scheme estimates the derivative as:

$$
f^{\prime}\left(x_{0}\right)=\lim _{\Delta x \rightarrow 0} \frac{f\left(x_{0}+\Delta x\right)-f\left(x_{0}\right)}{\Delta x} \approx \frac{f\left(x_{0}+h\right)-f\left(x_{0}\right)}{h},
$$

where a small perturbation $h \in \mathbb{R}$ is used to approximate $\lim _{\Delta x \rightarrow 0}(\cdot)$. It appears that the smaller $h$ is, the better approximate Eq. (1) delivers. However, we are not allowed to make $h$ arbitrarily small to improve the precision of Eq. (1). This is because the subtraction between two nearly equal numbers, such as $f\left(x_{0}+h\right)-f\left(x_{0}\right)$ in Eq. (1) when $h$ is very small, could eliminate many of their significant digits and contaminate the result. This issue is often known as the subtractive cancellation. During the simulation, finite difference would accumulate numerical errors along the time integration and crash the solver quickly.

Fortunately, this numerical instability can be averted using the so-called complex-step finite difference [Abreu et al. 2018; Martins et al. 2003; Squire and Trapp 1998] or CSFD. The trick is to apply the perturbation $h$ in the imaginary domain after promoting $f$ to be a complex function. The subtraction of the first-order terms is skipped in the complex Taylor expansion [Lyness 1968], and we can make the perturbation $h$ very small to accurately approximate the derivative without worrying about the subtractive cancellation. CSFD allows us to conveniently obtain a highly accurate numerical derivative without deriving its actual formulation, which could be otherwise tedious and error-prone.

On the downside, CSFD has several fundamental limitations. First of all, promoting a real-valued function to be a complex-valued one induces significant computation overheads. A naïve CSFD implementation as in existing literature [Martins et al. 2003; Squire and Trapp 1998] is often orders-of-magnitude slower than the analytic derivative. Secondly, complex-version Taylor expansion only circumvents the subtractive cancellation of the first-order derivative. Second- and higher-order derivatives still suffer with this issue and cannot be robustly approximated. In many simulation problems, we also need to deal with tensor functions, whose outputs are based on complicated numerical routines like Cholesky decomposition or SVD. Original CSFD becomes awkward as those numerical procedures are difficult to be explicitly formulated. Promoting such tensor functions is rather involving, if not impossible. As an echo to those drawbacks, we augment classic CSFD scheme making it more efficient, generalizable, and robust. While our extensions utilize some known techniques like multicomplex number [Fleury et al. 1993; Price 1991] and CauchyâĂŞRiemann equation [Ahlfors 1973], to the best of our knowledge, we are the first to optimize CSFD performance making it nearly as efficient as the analytic derivative, re-engineer it to be a handy off-the-shelf numerical differentiation solution for physics animation, and thoroughly validate its feasibility in the context of deformable simulation. Specifically, we would like to summarize our contributions as follows:

- Analysis CSFD is a relatively new numerical method. We provide an extensive explanation of its numerical mechanism, error source, and theoretical foundation.

- Acceleration We systematically optimize the original CSFD scheme. Without losing accuracy, we obtain multifold speedups, and our accelerated CSFD is as efficient as the analytic derivative. This is achieved by discarding high-order error terms, decoupling real and imaginary calculations, replacing expensive functions (e.g. trigonometric functions), and isolating the propagation of the perturbation in composite and nesting functions.

- Adaptation Instead of resorting to high-order Taylor expansion or Fourier expansion, we choose to promote a real-valued function with multicomplex arithmetic, which leads to a multicomplexstep finite difference scheme (MCSFD) for high-order finite difference. Our acceleration techniques naturally synergize with this generalization. In addition, we leverage the Cauchy-Riemann formula to further adapt CSFD/MCSFD for tensor functions.

- Application We thoroughly validate CSFD/MCSFD in both numerical experiments as well as in complicated nonlinear deformable simulations. Without knowing the actual formulation of the internal force and the tangent stiffness matrix, nonlinear deformation can be robustly and accurately simulated with CSFD/MCSFD. First- and high-order modal derivative bases can also be constructed for sophisticated materials. Many challenging inverse simulation problems now can be easily tackled using standard gradient/Hessian-based optimization approaches such as the Newton's method (e.g. see Fig. 1).

\section{RELATED WORK}

Calculating the differentiation of a function is an important computational procedure in many graphics research problems. For instance, in physics-based animation [Witkin 1997], such as rigid body dynamics [Baraff 1989, 1991], fluid/smoke animation [Bridson 2015], and cloth simulation [Baraff and Witkin 1998; Goldenthal et al. 2007], etc, the key challenge is to solve the unknown ordinary/partial differential equations, and one needs to use numerical approaches to discretize the differential operation. In computational fabrication, the optimal design is often obtained via following the gradient direction of the inverse simulation [Chen et al. 2014; Schulz et al. 2017; Yan et al. 2018], not to mention a vast volume of research involving various optimization procedures, many of which rely on the information of the gradient and/or Hessian of the objective function.

Evaluating the derivative is also a key ingredient in deformable simulation [Terzopoulos et al. 1987] especially for hyperelastic models [Bonet and Wood 1997]. Those materials are fully characterized by the strain energy density $E(\mathbf{F})$ of the local deformation gradient F. Modeling such materials requires the first- and/or second-order spatial derivatives of $E$ to establish the equilibrium equation. Dynamic simulation can also be casted as an optimization problem of the variational form [Liu et al. 2013; Stern and Desbrun 2006]. Newton's method [Capell et al. 2002], quasi-Newton method [Liu et al. 2017] or gradient descent method [Wang and Yang 2016] can then be used when gradient information is provided. The closed-form formulation of derivatives of $E$ for some materials can be found in the literature [Bonet and Wood 1997; Sifakis and Barbic 2012; Smith et al. 2018]. However, for other more complicated models like phenomenological and user-crafted materials [Koyama et al. 2012; Martin et al. 2011], obtaining the analytic derivative is non-trivial and labor-intensive. For principal stretch based nonlinear materials, such as Ogden and spline-based materials [Xu et al. 2015], careful 
numerical thresholding is required, even at the rest configuration, to obtain the actual tangent stiffness matrix. Deriving those derivatives analytically could be tedious and seemingly unworthy, if the user just wants to toy with a new hyperelastic energy to see how it behaves in a given animation scenario. Even with the help of symbolic differentiation packages like Mathematica [Wolfram et al. 1996] and Maple [Maple 1994], the implementation efforts are still considerable. Besides, there are also many cases where the target function's formulation is not even accessible, and one has to use the numerical derivative to infer the underlying kinematics [Barbič et al. 2012; Hahn et al. 2012, 2013]. In model reduction, it is known that linear modes are not sufficient to capture large nonlinear deformation, and the modal derivative [Barbič and James 2005; Yang et al. 2015] should be used. Those derivative modes are computed through evaluating the third-order gradient of the energy function (i.e. the Hessian of the internal force), which makes this technique less popular for more sophisticated materials other than the St. Venant-Kirchhoff (StVK) model.

The finite difference method is a standard procedure of computing the numerical derivative [Renardy and Rogers 2006]. Its variances include forward difference (FD), backward difference (BD), and central difference (CD). $\mathrm{CD}$ is twice as expensive as FD or $\mathrm{BD}$, but it is also the most accurate among them. Nevertheless, all of these schemes suffer from the subtractive cancellation issue: decreasing the magnitude of the perturbation does not make the finite difference converging, and the result will oscillate around the correct value and explode eventually [Brezillon et al. 1981]. This numerical behavior prevents the adoption of the finite difference method for applications that are sensitive to the accuracy of the differentiation.

On the other hand, CSFD is a powerful finite difference scheme but often overlooked in classic numerical analysis textbooks [Squire and Trapp 1998]. This method is based on the complex version of Taylor series expansion of a function, which dates back to the 1960s [Lyness 1967]. Unlike regular finite difference method, CSFD obviates the subtractive cancellation problem (in the first-order approximation) so that a very small perturbation (e.g. $1.0 \times 10^{-20}$ or even smaller) can be used making the resulting derivative approximation highly accurate. Indeed, we show that CSFD is able to completely replace the analytic gradient without any accuracy concerns in deformable simulation. Due to its superior accuracy, CSFD has been gradually recognized and used for the sensitivity analysis [Anderson et al. 2001; Butuk and Pemba 2003; Montoya et al. 2014; Voorhees et al. 2011]. For nonlinear finite element method (FEM) simulation with high-order shape functions, CSFD has also been used to obtain the numerical tangent stiffness matrix [Kim et al. 2011; Lebofsky 2013; Pérez-Foguet et al. 2000].

Because the target function is promoted to be a complex one, a naïve implementation of CSFD involves much heavier computations than the real-valued finite difference. We show that this limitation can be ameliorated by carefully manipulating the promoted target function and discarding high-order perturbation terms. Our results show that we are able to achieve a multifold speedup, making CSFD nearly as efficient as using the exact derivative. Instead of referring to the Fourier differentiation [Bagley 2006; Lai and Crassidis 2008], we use the multicomplex-step finite difference [Lantoine et al.
2012] to handle high-order derivative. Doing so allows our acceleration scheme to be seamlessly integrated for high-order numerical derivatives.

CSFD vs. automatic differentiation. Another relevant and widely known differentiation technique is the automatic differentiation (AD) [Griewank and Walther 2008; Nocedal and Wright 2006; Rall 1981], which decomposes complicated functions with the chain rule. $\mathrm{AD}$ has been used in graphics [Grinspun et al. 2003; Guenter 2007; Mitchell and Hanrahan 1992]. Indeed, the back propagation optimization [Hecht-Nielsen 1992] commonly used for neural network training is a special implementation of the reverse $A D$.

A key difference between CSFD and AD lies in the fact that " $A D$ uses exact formulas along with floating-point values" [Neidinger 2010], and it is "not numerical differentiation" [Baydin and Pearlmutter 2014]. CSFD, on the other hand, is a numerical approach seeking for the derivative approximation. AD is more sensitive to the smoothness of the function and could fail at discontinuities. CSFD behaves more robustly in such cases: because the complex perturbation is orthogonal to the real domain, CSFD always returns the derivative as long as the target function exists. $\mathrm{AD}$ also has practical difficulties for high-order generalization [Margossian 2018]. For instance, some existing AD packages (e.g. Adept [Hogan 2014]) only deals with the first-order derivative. While one may perform firstorder differentiation multiple times to obtain a high-order derivative, it has been argued that recursively applying $\mathrm{AD}$ leads to inefficient and numerically unstable code [Betancourt 2018; Margossian 2018]. High-order AD is seldom well supported and could be extremely slow. On the other hand, MCSFD extension generalizes our acceleration scheme to high-order cases with excellent robustness and accuracy. Our accelerated CSFD/MCSFD is over $30 \times$ faster than commonly used $\mathrm{AD}$ packages even for the first-order case. Tensor functions that involving complicated numerical procedures are also problematic with $\mathrm{AD}$. It remains unclear if the CauchyâĂŞRiemann generalization [Ahlfors 1973] can be applied in AD. Our accelerated CSFD is orthogonal to and complements the AD technique. Because CSFD is highly accurate (as accurate as the analytic result), it is possible to harness CSFD/MCSFD for calculating derivatives along the chain rule that could be otherwise troublesome to $\mathrm{AD}$.

\section{BACKGROUND}

In order to make the paper more self-contained, we start our discussion with a brief review of the error source of the finite difference method and the numerical issue of the subtractive cancellation.

Suppose that the function $f: \mathbb{R} \rightarrow \mathbb{R}$ is differentiable around $x=x_{0}$. After a small perturbation $h$ is applied, it can be Taylor expanded as:

$$
\begin{aligned}
f\left(x_{0}+h\right) & =f\left(x_{0}\right)+f^{\prime}\left(x_{0}\right) \cdot h+\frac{1}{2} f^{\prime \prime}\left(x_{0}\right) \cdot h^{2}+\cdots \\
& =f\left(x_{0}\right)+f^{\prime}\left(x_{0}\right) \cdot h+\mathbf{O}\left(h^{2}\right),
\end{aligned}
$$

which leads to the forward finite difference of Eq. (1). Eq. (2) also suggests that $h$ should be as small as possible for a good approximation. In the meantime, because the total number of bits used to represent a real number is limited on a computer, all the floatingpoint arithmetics have the round-off error [Ueberhuber 2012], which 
is a small relative error also known as machine epsilon $\epsilon$. For the double precision of IEEE 754 floating-point standard [IEEE 1985], $\epsilon \approx 1.11 \times 10^{-16}$. Normally, the round-off error does not seriously impair the stability or the accuracy of a numerical procedure. However, when $h$ gets smaller, $f\left(x_{0}+h\right)$ and $f\left(x_{0}\right)$ become nearly equal to each other. Subtraction between them would eliminate many significant digits, and the result after rounding could largely deviate from the actual value of $f\left(x_{0}+h\right)-f\left(x_{0}\right)$.

We elaborate this issue using a simple four-digit decimal floatingpoint system. Here, a real number $a=1999.99$ is represented as $\widetilde{a}=1.999 \times 10^{3}$ (because we only have four digits for the mantissa), and we use $\widetilde{(\cdot)}$ to denote a digitalized number in a floating-point system. In this example, we simply choose the round-by-chop rule that discards all the out-of-precision digits, and the corresponding round-off error is:

$$
E_{\text {round }}=\frac{|a-\tilde{a}|}{|a|}=\frac{\left|1999.99-1.999 \times 10^{3}\right|}{|1999.99|} \approx 4.95 \times 10^{-4} .
$$

Next, let $b=1998.88$, which is represented as $\widetilde{b}=1.998 \times 10^{3}$. The error of calculating $a-b$ with this toy floating-point system is:

$$
\begin{aligned}
E_{\text {subtraction }} & =\frac{|(\widetilde{a}-\tilde{b})-(a-b)|}{|a-b|} \\
& =\frac{\left|(1.999-1.998) \times 10^{3}-(1999.99-1998.88)\right|}{|1999.99-1998.88|} \\
& \approx 0.1 .
\end{aligned}
$$

We can see from Eqs. (3) and (4) that rounding loses us the least important significant digit, and it only yields an error at the order of the floating-point precision $\left(10^{-4}\right)$. However, the subtraction between $\widetilde{a}$ and $\widetilde{b}$ eliminates three leading significant digits, which yields a much more substantial error. If we set $b$ even closer to $a$ as $b=1999.88, E_{\text {subtraction }}$ increases to $100 \%$ as all the significant digits are eliminated. This is why the cancellation of subtracting numbers of similar magnitude is also called catastrophic cancellation.

Some numerical literature (e.g. [Nocedal and Wright 2006]) shows that $\mathrm{CD}$ with the form of:

$$
f^{\prime}\left(x_{0}\right) \approx \frac{f\left(x_{0}+h\right)-f\left(x_{0}-h\right)}{2 h},
$$

has a better accuracy with a quadratic error, while FD and BD have an error term of $\mathbf{O}(h)$. This conclusion is based on the assumption that subtractive cancellation does not occur. As to be discussed in the next section, CD could be even more sensitive to a smaller $h$ (because of its faster convergent rate).

\section{COMPLEX-STEP FINITE DIFFERENCE}

CSFD is based on the complex Taylor series expansion [Lyness 1968]. Let $(\cdot)^{*}$ denote a complex variable, and suppose $f^{*}: \mathbb{C} \rightarrow \mathbb{C}$ is differentiable around $x_{0}^{*}=x_{0}+0 i$. If a perturbation $h$ is applied at the imaginary domain, $f^{*}$ can be expanded as:

$$
f^{*}\left(x_{0}+h i\right)=f^{*}\left(x_{0}^{*}\right)+f^{*^{\prime}}\left(x_{0}^{*}\right) \cdot h i+\mathbf{O}\left(h^{2}\right) .
$$

For any smooth and real-valued function $f$, we can always lift it to be a complex one $f^{*}$ by allowing complex input while retaining its computation procedure unchanged. Under this circumstance, both $f^{*}\left(x_{0}^{*}\right)=f\left(x_{0}\right) \in \mathbb{R}$ and $f^{*^{\prime}}\left(x_{0}^{*}\right)=f^{\prime}\left(x_{0}\right) \in \mathbb{R}$ do not have

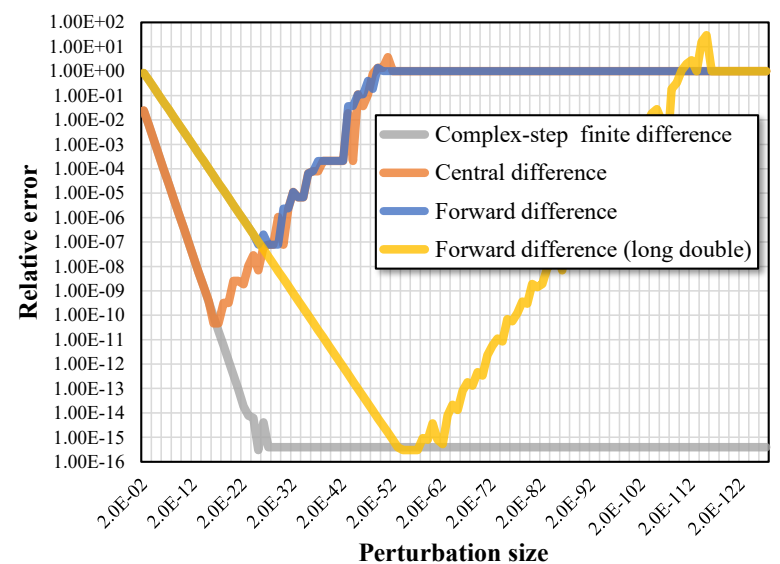

Fig. 2. We use FD, CD, and CSFD to calculate the first-order derivative of $f(x)=e^{x} /\left(x^{4}+x^{2}+1\right)$ at $x=4$. The resulting numerical derivative is compared with the analytic derivative, and the relative error is plotted against the size of the perturbation, ranging from $2^{-2}$ to $2^{-127}$.

imaginary parts. Taking the imaginary part (i.e. using the operator $\operatorname{Im}(\cdot) \in \mathbb{R})$ of both sides of Eq. (6) leads to $\operatorname{Im}\left(f^{*}\left(x_{0}+h i\right)\right)=$ $\operatorname{Im}\left(f^{*}\left(x_{0}^{*}\right)+f^{*^{\prime}}\left(x_{0}^{*}\right) \cdot h i\right)+\mathbf{O}\left(h^{3}\right)$. We can then have the first-order CSFD approximation:

$$
f^{\prime}\left(x_{0}\right)=\frac{\operatorname{Im}\left(f^{*}\left(x_{0}+h i\right)\right)}{h}+\mathbf{O}\left(h^{2}\right) \approx \frac{\operatorname{Im}\left(f^{*}\left(x_{0}+h i\right)\right)}{h} .
$$

Compared with Eq. (1) or Eq. (5), we can see that Eq. (7) does not have a subtractive numerator meaning it only has the round-off error regardless of the size of the perturbation $h$. In addition, the operation of $\operatorname{Im}(\cdot)$ removes the $(h i)^{2}$ term in Eq. (6), making the actual approximation error $\mathbf{O}\left(h^{2}\right)$. Thus, we can employ a very small $h$ to obtain a highly accurate numerical derivative.

In addition to complex-step finite difference of Eq. (7), it is also possible to apply the perturbation in the dual domain, which corresponds to the dual number method [Fike and Alonso 2011; Fike 2013]. Similar to the complex number, a dual number $d=a+b \epsilon$ has a real part $a$ and a dual part $b \epsilon$ such that $\epsilon \neq 0$ but $\epsilon^{2}=0$. This property makes all the higher-order terms of $\epsilon$ vanished. As a result, the dual version Taylor expansion of a given function leads to $f\left(x_{0}+h \epsilon\right)=f\left(x_{0}\right)+f^{\prime}\left(x_{0}\right) \cdot h \epsilon$, and one can obtain the exact derivative by exacting dual part of $f\left(x_{0}+h \epsilon\right)$ and setting $h=1$ as: $f^{\prime}\left(x_{0}\right)=\operatorname{Du}\left(f\left(x_{0}+\epsilon\right)\right)$. The question here is how can we evaluate the dual function $f\left(x_{0}+\epsilon\right)$. The literature of dual number arithmetic is far less extensive than the complex arithmetic. Normally, a dual function can be evaluated as a dual polynomial [Kramer 1930], which is based on the analytic derivative $f^{\prime}\left(x_{0}\right)$. In other words, the dual number formulation is equivalent to using the analytic differentiation (because you need to compute $f^{\prime}\left(x_{0}\right)$ to obtain the promoted dual function).

Fig. 2 reports a numerical experiment of $f(x)=e^{x} /\left(x^{4}+x^{2}+1\right)$. We compute the first-order numerical derivative at $x=4$ using FD, $\mathrm{CD}$ and CSFD. The analytic derivative of this simple function can be derived as: $f^{\prime}(x)=\left(x^{4}-4 x^{3}+x^{2}-2 x+1\right) e^{x} /\left(x^{4}+x^{2}+1\right)^{2}$, and $f^{\prime}(4)=0.006593183194438$ is considered as the ground truth. In this 
example, both $f(x)$ and $f^{\prime}(x)$ are well scaled, and the issue of subtractive cancellation starts to take place when $h \approx 2^{-26} \sim 1.0 \times 10^{-8}$ with FD and $h \approx 2^{-17} \sim 1.0 \times 10^{-6}$ with $\mathrm{CD}$. We do see CD converges faster than FD before hitting the threshold of subtractive cancellation. However, both CD and FD explode quickly after the cancellation occurs. When $h$ becomes smaller than $2^{-47} \sim 1.0 \times 10^{-15}$, the subtractive cancellation eliminates all the significant digits making $f\left(x_{0}+h\right)-f\left(x_{0}\right)$ and $f\left(x_{0}+h\right)-f\left(x_{0}-h\right)$ vanished by the rounding. In this case, we cannot obtain any useful information of the derivative out of the finite difference approximation, and the relative error stays $100 \%$. The numerical performance of FD can be improved by extending the floating number precision. As indicated in the figure, after doubling the precision from double to long double (128 bit), the subtractive cancellation is delayed. If we choose the perturbation size carefully, FD is also able to yield good accuracy in this particular example. In real applications however, $f$ often takes a high-dimension vector $\mathbf{x}$ as the dependable variable. $f$ and $\partial f / \partial x_{i}$ may also be badly scaled. These circumstances make subtractive cancellation happen much earlier. As a result, the numerical derivative of FD and CD is fallible: a conservative $h$ has a big approximation error, while an aggressive $h$ could be even worse due to the cancellation. In physics-based simulations, $\mathrm{FD} / \mathrm{CD}$ is always problematic and often the demon behind the numerical instability. On the other hand, CSFD shows a superior performance in terms of both convergence rate and numerical stability. As CSFD does not have the subtractive cancellation problem, the relative error decreases consistently with a smaller $h$. When $h$ is sufficiently small (i.e. $h<2^{-26} \sim 1.0 \times 10^{-8}$ ), CSFD delivers a result with an error below $1.0 \times 10^{-15}$. Note that the "ground truth" itself also has a round-off error at the order of $10^{-16}$. In other words, CSFD is as accurate as the analytic derivative for a sufficiently small $h$.

Naïve complex promotion. In order to apply CSFD, we must promote the real function $f(x)$ to be a complex one $f^{*}\left(x^{*}\right)$. While the specific form of $f(x)$ could be complicated, it is always constructed with binary operators of,,$+- \times, \div$ and unary operators including power function $\left(x^{a}\right)$, exponential function $\left(e^{x}\right)$, logarithmic function $(\ln x)$, and trigonometric functions ( $\sin x$ etc.). Promoting these elementary functions follows the standard complex number arithmetic [Ablowitz and Fokas 2003]. For a quick reference, we also list the complex promotion of some commonly used functions in Appendix A.

If efficiency is not the primary concern, CSFD can be quickly implemented via overloading existing floating-point arithmetic operators with the corresponding complex version. C++ Template provides a flexible mechanism for this purpose: one can code $f(x)$ using a generic data type and choose the complex-type template specialization when CSFD is needed. Standard C++ STD library has a collection of stable complex number routines. Besides, there are also a few third-party opensource complex number libraries such as Boost [Schäling 2011] and Eigen [Guennebaud et al. 2014]. Nevertheless, such naïve CSFD implementation induces a significant overhead. In many cases, CSFD runs orders-of-magnitude slower than the analytic derivative. One of our major contributions is to optimize CSFD computation to regain the efficiency of the finite difference. This is to be detailed in the next section.

\section{CSFD ACCELERATION}

Using general-purpose complex number arithmetic to promote $f(x)$ is actually "overkill" for just using CSFD to compute numerical derivatives. We show that CSFD approximation can be substantially simplified and accelerated, and our accelerated CSFD is as efficient as using the analytic derivative. Our strategy is based on the following three important observations:

- According to Eq. (7), it is clear that calculating the real part of $f^{*}$ is unnecessary for CSFD, therefore nearly half of the computation brought by the complex promotion can be discarded.

- Complex number arithmetic for CSFD is quite different from a general complex operation. The imaginary part of $f^{*}$ comes from the applied perturbation $h i$, which is a very small value (i.e. $\left.h<1.0 \times 10^{-20}\right)$. Many calculations can be simplified by treating $h$ as an infinitesimal: for instance we can have $\sin h \sim h$ to avoid the expensive evaluation of the trigonometric function of $\sin h$.

- Because $h$ appears as the denominator of Eq. (7), all the quadratic or higher-order terms of $h$ in $\operatorname{Im}\left(f^{*}\left(x_{0}+h i\right)\right)$ can be discarded, which only leads to an approximation error up to $\mathbf{O}(h)$.

\subsection{Accelerate CSFD of a Single Elementary Function}

We start our discussion by assuming that $f(x)$ is an elementary function (i.e. listed in Appendix A), and take $f(x)=x^{1 / m}$ an example to show how it can be much more efficiently evaluated for CSFD. First, the standard complex promotion (Eq. (44)) gives us:

$$
\frac{\operatorname{Im}\left(f^{*}\left(x_{0}+h i\right)\right)}{h}=\frac{1}{h}\left(r^{\frac{1}{m}} \cdot \sin \frac{\phi}{m}\right) .
$$

Here, $r(\cos \phi+\sin \phi i)$ is the polar form of $x_{0}+h i$. Recalling that $h$ is a very small quantity, we have:

$$
\sin \phi=\frac{h}{r} \Rightarrow \phi=\frac{h}{r}
$$

because $\langle\sin a \sim a\rangle$ is a pair of equivalent infinitesimals when $a \rightarrow 0$. With Eq. (9), the RHS of Eq. (8) can be greatly simplified as:

$$
\frac{1}{h}\left(r^{\frac{1}{m}} \cdot \sin \frac{\phi}{m}\right)=\frac{1}{h}\left(r^{\frac{1}{m}} \cdot \frac{\phi}{m}\right)=\frac{1}{h}\left(r^{\frac{1}{m}} \cdot \frac{h}{r m}\right)=\frac{r^{\frac{1}{m}}}{r m} .
$$

Table 1. Time statistics of using the optimized CSFD formulations (i.e. Eqs. (8) and (10)) and the naïve CSFD implementation (Eq. (44)) of the exponential function $f(x)=x^{1 / m}$ for 100 million times. The computation time using analytic derivative is also reported for the reference. Our CSFD simplification is over $200 \times$ faster than the naïve implementation. In this example, it is even faster than using the analytical derivative. The relative error is at the order of the machine epsilon $\left(10^{-16}\right)$.

\begin{tabular}{c|c|c|c}
\hline Eq. (44) & Eq. (8) & Eq. (10) & Analytic \\
\hline $13.1 s$ & $9.49 s(1.4 \times)$ & $0.056 s(233 \times)$ & $0.064 s$ \\
\hline
\end{tabular}

The performance improvement of Eq. (10) is substantial. We record the computation time of running Eqs. (44), (8), and (10) respectively as well as directly calculating the analytical derivative of $\left(x^{\frac{1}{m}}\right)^{\prime}=\frac{1}{m} x^{\frac{1}{m}-1}$ for 100 million times on an Intel i 7 laptop. The result is reported in Tab. 1. As expected, we can see from the table 


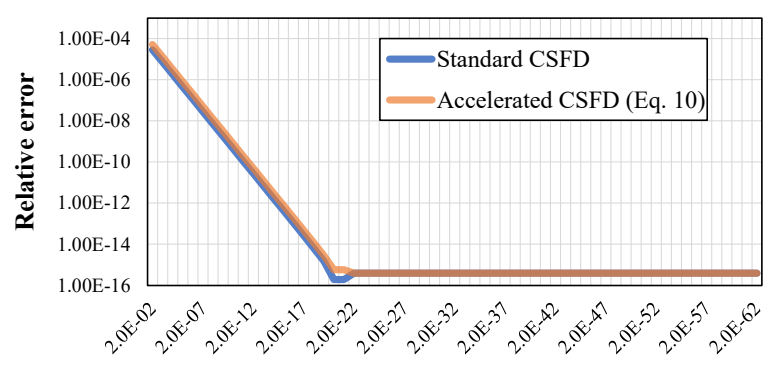

Perturbation size

Fig. 3. Our fast CSFD implementation has good numerical stability and accuracy. The relative error converges as quickly as the regular CSFD and remains at the order of machine epsilon after $h$ is sufficiently small.

that Eq. (8) modestly improves the calculation efficiency by discarding real part computation. The most significant speedup originates from equivalent infinitesimal based simplification, which frees us from performing expensive trigonometric function calculation. In this example, CSFD is even faster than using analytic derivative because Eq. (10) has a simpler exponential term of $(\cdot)^{\frac{1}{m}}$ than the exponential term in the analytic derivative: $(\cdot)^{\frac{1}{m}-1}$. Meanwhile, our accelerated CSFD retains all the favored advantages of CSFD. As shown in Fig. 3, fast CSFD implementation has the same convergency and accuracy. For small $h$, the relative error reaches the machine epsilon stably. Interestingly, if one further simplifies $r$ such that $r=\sqrt{x_{0}^{2}+h^{2}}=x_{0}$ when $h \rightarrow 0$, Eq. (10) converges to the analytic derivative formulation. This finding reveals that, unlike regular finite difference, the actual derivative of the function is essentially hidden in its complex promotion. This is another important reason that explains why CSFD is able to achieve such high accuracy.

The strategy of leveraging equivalent infinitesimals can be readily applied to other elementary functions. For instance for trigonometric functions, the most expensive arithmetic is the evaluation of $e^{ \pm h}$. Again, because $h$ is an infinitesimal, we exploit the fact that $\left\langle e^{ \pm h} \sim 1 \pm h\right\rangle$ is also a pair of equivalent infinitesimals. This simplification brings another orders-of-magnitude speedup.

\subsection{Accelerate CSFD of Composite Binary Operators}

In reality, $f(x)$ houses a chain of binary operators such that:

$$
f(x)=f_{1}(x) \circ f_{2}(x) \circ f_{3}(x) \circ \cdots \circ f_{k}(x) \circ \cdots \circ f_{N}(x),
$$

for $\circ \in\{+,-, \times, \div\}$. Each $f_{k}(x)$ could also be a nesting composite of multiple unary functions: $f_{k}(x)=f_{k, 1}\left(f_{k, 2}\left(f_{k, 3}(\ldots)\right)\right)$. We defer the discussion of nesting operators to the next subsection and assume that the promoted form of each function along the chain is known.

Eq. (11) may be split into several sub-chains according to the parenthesization and operator priority. For instance, the example used in Fig. 2 can be understood as $f(x)=f_{1}(x) /\left(f_{2}(x)+f_{3}(x)+\right.$ $f_{4}(x)$ ), where $f_{1}(x)=e^{x}$ is an exponential function; $f_{2}(x)=x^{4}$ and $f_{3}(x)=x^{2}$ are power functions; and $f_{4}(x)=1$ is a constant. If a sub-chain only consists of addition and subtraction operators, which are independent for real and imaginary parts, we just evaluate the

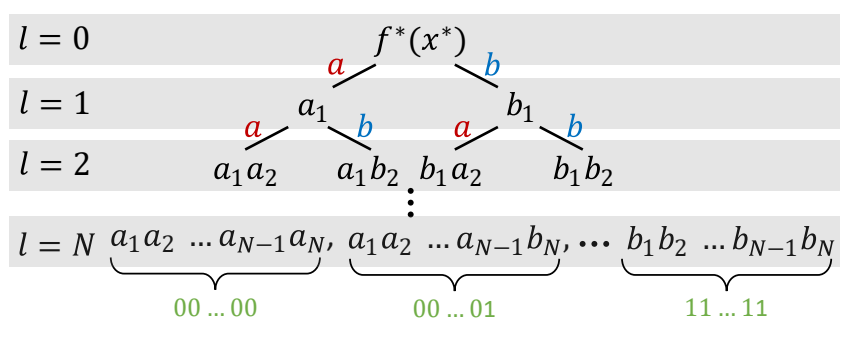

Fig. 4. The procedure of evaluating a chain of multiplications (and divisions) can be visualized with a binary tree. The leaf nodes can be concisely encoded by a binary number. As a result, we can discard higher-order infinitesimals with two or more 1s (e.g. 011) at the bottom level.

imaginary part of each promoted function $f_{k}^{*}$ along the chain for CSFD approximation and ignore the calculation for the real part.

However, if the sub-chain is concatenated with multiplication and/or division operators, we cannot discard the real part of each function because the real and imaginary parts are coupled in the multiplication operation - one can easily verify that the imaginary part of $f_{1}^{*}\left(x^{*}\right) \cdot f_{2}^{*}\left(x^{*}\right)$ contains the information of both real and imaginary parts of $f_{1}^{*}\left(x^{*}\right)$ and $f_{2}^{*}\left(x^{*}\right)$. Division is similar, which is regarded as the multiplication of the conjugate of the dividend.

We show that evaluating a multiplication chain can also be significantly accelerated based on a binary branching strategy. Let $f_{k}^{*}\left(x^{*}\right)=a_{k}+b_{k}$ denote a promoted function on the chain, where $b_{k}$ is an imaginary quantity. Our base case is the chain of a single promoted function $f^{*}\left(x^{*}\right)=f_{1}^{*}\left(x^{*}\right)=a_{1}+b_{1}$ with two addends. Putting an additional multiplying function after it leads to $f^{*}\left(x^{*}\right)=f_{1}^{*}\left(x^{*}\right) \cdot f_{2}^{*}\left(x^{*}\right)=\left(a_{1}+b_{1}\right) a_{2}+\left(a_{1}+b_{1}\right) b_{2}$. In other words, each item of $a_{1}$ and $b_{1}$ is multiplied by $a_{2}$ and $b_{2}$ respectively. The multiplication of $f_{2}^{*}\left(x^{*}\right)$ thus doubles the total number of addends. This procedure can also be visualized with a binary tree shown in Fig. 4. Each complex function $f_{k}^{*}\left(x^{*}\right)$ along the chain increments the height of the tree by one, and we have $2^{N}$ addends at the bottom level for a chain of $N$ functions. Recall that imaginary parts of $b_{k}$ correspond to a very small perturbation $b_{k}=h i \sim 0$, and we can discard all addends that are quadratic or higher-order of $b_{k}$. The key question here is how can we directly identify those addends without actually expanding the multiplication chain?

From Fig. 4, we can see that each extra multiplication induces a binary branch towards the next level. A left branch appends an $a_{k}$ after an existing addend while a right branch appends a $b_{k}$. The final form of a leaf addend depends on how many left and right branches at which levels it takes along the path from the root. Clearly, the leftmost and rightmost leaves are always $a_{1} a_{2} \ldots a_{N-1} a_{N}$ and $b_{1} b_{2} \ldots b_{N-1} b_{N}$. The second leftmost leaf differs from the leftmost one because it takes a right branch at the last level. Accordingly, its final form becomes $a_{1} a_{2} \ldots a_{N-1} b_{N}$. Interestingly, this branching mechanism mimics the ripple-carry addition of binary numbers. If we encode $a_{k}$ with 0 and $b_{k}$ with 1 , all the addends at the bottom level, from left to right, can be concisely represented as a sequence of binary numbers $B_{0}, B_{1}, B_{2}, \ldots, B_{2^{N-1}}$ such that $B_{k}=(k)_{\text {binary }}$ is the binary representation of the decimal index $k$. For instance, if we have three functions along the chain, the eight leaf addends from $B_{0}$ to $B_{7}$ are: $000,001,010,011,100,101,110$ and 111 . The number of 
ones in $B_{k}$ implies the order of $h$. Since anything higher-order than $h^{2}$ can be safely discarded, we only sum up addends with exact one 1-digit (the leftmost leaf is a real number, which is also discarded) such that: $\operatorname{Im}\left(f^{*}\left(x^{*}\right)\right)=a_{1} a_{2} b_{3}+a_{1} b_{2} a_{3}+b_{1} a_{2} a_{3}+\mathbf{O}\left(h^{2}\right)$. From Eq. (6), we can also understand that $f\left(x_{0}\right)=\operatorname{Re}\left(f^{*}\left(x_{0}+h i\right)\right)+\mathbf{O}\left(h^{2}\right)$ meaning replacing $a_{k}$ by $f_{k}(x)$ only induces an approximation error of $\mathbf{O}\left(h^{2}\right)$. As a result, we can stick with our acceleration strategy of ignoring the real part of a promoted function. If a long multiplication chain is identified (e.g. more than 10 multiplicands) whose derivative is to be evaluated via CSFD, we also pre-compute the product among all the $a_{k}$ as:

$$
A=\Pi_{k=1}^{N} a_{k}=\Pi_{k=1}^{N} f_{k}(x)+\mathbf{O}\left(h^{2}\right) .
$$

Therefore, a leaf node, say $a_{1} b_{2} a_{3}$ for instance, can be efficiently computed at $\mathbf{O}(1)$ time as:

$a_{1} b_{2} a_{3}=\operatorname{Re}\left(f_{1}^{*}\left(x^{*}\right)\right) \cdot \operatorname{Im}\left(f_{2}^{*}\left(x^{*}\right)\right) \cdot \operatorname{Re}\left(f_{3}^{*}\left(x^{*}\right)\right) \approx \frac{A}{f_{2}(x)} \cdot \operatorname{Im}\left(f_{2}^{*}\left(x^{*}\right)\right)$.

Note that using Eq. (12) potentially brings us the division-by-zero issue if $f_{k}(x)$ is zero or close to zero. This risk can be avoided by rolling back to the standard formula of $a_{1} b_{2} a_{3}$ instead of Eq. (13), if $f_{2}(x)$ is found smaller than a given threshold (say $1.0 \times 10^{-16}$ ). The timing benchmark shows that our strategy brings CSFD approximation an additional $5 \times$ boost. After the value of each $f_{k}(x)$ is computed, the naïve implementation uses $21 \mathrm{~ms}$ to calculate the first-order CSFD derivative for $N=100$, while our method only needs $4 \mathrm{~ms}$ on an $i 7$ laptop.

\subsection{Accelerate CSFD of Composite Unary Operators}

Real-world functions may also be in a nesting form of multiple unary operators:

$$
f(x)=f_{N}\left(f_{N-1}\left(f_{N-2}\left(\cdots f_{2}\left(f_{1}(x)\right)\right)\right)\right),
$$

where each $f_{k}$ for $1 \leq k \leq N$ could be a power, exponential, logarithmic, or trigonometric function. We stick with the notation of $f_{k}^{*}\left(x^{*}\right)=a_{k}+b_{k}$, where $b_{k}$ is an imaginary quantity, and $x_{0}+h i=a_{0}+b_{0}$ is the input of $f_{1}^{*}$ i.e. the innermost function. Note that the CSFD approximation of $f^{\prime}(x)$ is actually the ratio between $b_{N}$ and $b_{0}$ :

$$
f^{\prime}(x) \approx \frac{\operatorname{Im}\left(f_{N}^{*}\right)}{h}=\frac{\operatorname{Im}\left(f_{N}^{*}\right) i}{h i}=\frac{\operatorname{Im}\left(a_{N}+b_{N}\right) i}{h i}=\frac{b_{N}}{b_{0}} .
$$

Similar to the multiplication case, the real and imaginary parts of an outer function are also coupled with the input real and imaginary parts from its inner function. The algebraic relation between $b_{N}$ and $b_{0}$ could be complicated, and expanding the entire composite equation to compute the actual value of $b_{N}$ is expensive.

Fortunately we notice that in order to compute $f^{\prime}(x)$ with CSFD, only the ratio between $b_{N}$ and $b_{0}$ is needed, and their exact values are of less interest. Therefore, we convert $b_{N} / b_{0}$ to be:

$$
\frac{b_{N}}{b_{0}}=\frac{b_{1}}{b_{0}} \cdot \frac{b_{2}}{b_{1}} \cdot \frac{b_{3}}{b_{2}} \cdot \ldots \cdot \frac{b_{N}}{b_{N-1}} .
$$

A multiplicand in RHS of Eq. (16), $b_{k} / b_{k-1}$, describes how the imaginary perturbation is changed through $f_{k}^{*}$. An important observation here is the imaginary part of a promoted function remains infinitesimally small after being applied with an infinitesimal imaginary perturbation. This can be easily verified by the complex Taylor expansion: $f^{*}\left(x_{0}+h i\right) \approx f^{*}\left(x_{0}^{*}\right)+f^{*^{\prime}}\left(x_{0}^{*}\right) \cdot h i$, which leads to $\operatorname{Im}\left(f^{*}\left(x_{0}+h i\right)\right) \approx f^{\prime}\left(x_{0}\right) \cdot h=\mathbf{O}(h) \sim h$. In other words, all the $b_{k}$ in Eq. (16) are small imaginary perturbations of the same order of $h i$. Therefore, we re-set each intermediate perturbation of $b_{k-1}$ as $h$. In the meantime, its real part input $a_{k-1}$ can be efficiently computed as $f_{k-1}$ without resorting to $f_{k-1}^{*}$ as:

$$
\begin{aligned}
\frac{b_{k}}{b_{k-1}} & =\frac{\operatorname{Im}\left(f_{k}^{*}\left(a_{k-1}+b_{k-1}\right)\right) i}{b_{k-1}} \\
& \approx \frac{\operatorname{Im}\left(f_{k}^{*}\left(a_{k-1}+h i\right)\right)}{h} \approx \frac{\operatorname{Im}\left(f_{k}^{*}\left(f_{k-1}+h i\right)\right)}{h} .
\end{aligned}
$$

Eq. (17) literally breaks the coupling of the imaginary parts along the nesting chain - when computing $b_{k} / b_{k-1}$, the actual imaginary values from inner functions are not required, and the propagation of the initial imaginary perturbation $b_{0}=h i$ is isolated.

Discussion. Eq. (16) should look immediately similar to the chain rule, which forms the foundation of $\mathrm{AD}$ techniques. Indeed, one may also understand Eq. (17) as breaking Eq. (14) using the chain rule and applying CSFD to approximate each intermediate derivative afterwards (i.e. by setting $b_{k-1}=h i$ and $a_{k-1}=f_{k-1}$ ). In other words, Eq. (16) is practically equivalent to augmenting $A D$ with accelerated $C S F D$ without referring to differentiation rules. Regular AD packages (e.g. CppAD [Bell 2012] and Adept [Hogan 2014]) mainly aim on first- or second-order derivatives, and their generalization to highorder derivative is nonintuitive and inefficient, if not impossible. However, as we will see in the next section, CSFD can be elegantly generalized to handle high-order derivatives. All the acceleration techniques discussed in this section are naturally inherited.

\section{MULTICOMPLEX-STEP PERTURBATION}

Regular finite difference evaluates high-order derivative by recursively applying the first-order approximation of Eq. (1). For instance, the second-order derivative is approximated as:

$$
f^{\prime \prime}\left(x_{0}\right)=\frac{f\left(x_{0}+h\right)-2 f\left(x_{0}\right)+f\left(x_{0}-h\right)}{h^{2}}+\mathbf{O}\left(h^{2}\right),
$$

which requires two extra function evaluations for both $f\left(x_{0}+h\right)$ and $f\left(x_{0}-h\right)$. The complex Taylor series expansion of Eq. (6) gives a real second-order term (with a factor of $i^{2}$ ), which yields:

$$
f^{\prime \prime}\left(x_{0}\right)=\frac{2\left(f\left(x_{0}\right)-\operatorname{Re}\left(f^{*}\left(x_{0}+h i\right)\right)\right)}{h^{2}}+\mathbf{O}\left(h^{2}\right) .
$$

Eq. (19) only needs one extra function evaluation of $f^{*}\left(x_{0}+h i\right)$ : its imaginary part can be used for the first-order CSFD while its real part is being used for the second-order CSFD. However, both schemes suffer with the subtractive cancellation. Besides, computing $f^{*}\left(x_{0}+\right.$ $h i)$ could be even slower than computing both $f\left(x_{0}+h\right)$ and $f\left(x_{0}-h\right)$ due to the extra complexity induced by the promotion. Therefore, second-order CSFD is less appealing to us. A more numerical stable approach is based on Fourier differentiation [Bagley 2006], which generalizes the complex Taylor expansion to Fourier expansion by 
not retaining the perturbation on the imaginary axis:

$$
f^{*}\left(x_{0}+h e^{\theta i}\right)=f^{*}\left(x_{0}^{*}\right)+f^{*^{\prime}}\left(x_{0}^{*}\right) \cdot h e^{\theta i}+f^{*^{\prime \prime}}\left(x_{0}^{*}\right) \cdot \frac{h^{2}}{2} e^{2 \theta i} \ldots .
$$

High-order derivative can be computed by using different argument angles of $\theta$ to cancel out unwanted terms. For instance, setting $\theta=\pi / 4$ and $\pi+\pi / 4$ leads to one possible second-order approximation [Lai and Crassidis 2008]:

$$
f^{*^{\prime \prime}}\left(x_{0}^{*}\right)=\frac{\operatorname{Im}\left(f^{*}\left(x_{0}+h \cdot i^{\frac{1}{2}}\right)+f^{*}\left(x_{0}-h \cdot i^{\frac{1}{2}}\right)\right)}{h^{2}}+\mathbf{O}\left(h^{2}\right) .
$$

While Fourier differentiation may be able to avoid the subtractive cancellation with a carefully chosen $\theta$, its formulation is quite different from the first-order CSFD ${ }^{1}$. In practice, users have to use distinct implementations for different differentiation orders, and most calculations among them cannot be shared.

Alternatively, there is a more concise formula that generalizes the perturbation to be a multicomplex quantity, and we refer to this method as multicomplex-step finite difference (MCSFD). The detailed derivation of MCSFD formulation can be found in existing literature [Lantoine et al. 2012; Nasir 2013]. MCSFD extends the regular complex number to have multiple mutual-orthogonal imaginary directions. The most attractive feature of multicomplex number to us is it can be defined recursively: its base cases are the real number $\mathbb{R}$ and the regular complex number $\mathbb{C}$, which are considered as zeroand first-order multicomplex sets $\mathbb{C}^{0}$ and $\mathbb{C}^{1}$. $\mathbb{C}^{1}$ extends the real set $\left(\mathbb{C}^{0}\right)$ by adding an imaginary unit $i$ as: $\mathbb{C}^{1}=\left\{x+y i \mid x, y \in \mathbb{C}^{0}\right\}$, and the multicomplex number up to an order of $n$ is defined as:

$$
\mathbb{C}^{n}=\left\{z_{1}+z_{2} i_{n} \mid z_{1}, z_{2} \in \mathbb{C}^{n-1}\right\} .
$$

The order of a multicomplex number matches the number of its imaginary directions, and all the imaginary units $i_{n}$ have the property of $i_{n}^{2}=-1$. Fully expanding the recurrence of Eq. (22) yields:

$$
\begin{aligned}
\mathbb{C}^{n}= & x_{0}+x_{1} i_{1}+x_{2} i_{2}+\cdots+x_{n} i_{n} \\
& +x_{1,2} i_{1} i_{2}+\cdots+x_{n-1, n} i_{n-1} i_{n} \\
& +x_{1,2,3} i_{1} i_{2} i_{3}+\cdots+x_{n-2, n-1, n} i_{n-2} i_{n-1} i_{n} \\
& \vdots \\
& + \\
& +x_{1,2, \ldots, n} i_{1} i_{2} \cdots i_{n},
\end{aligned}
$$

where all of $x_{0}, x_{1}, \ldots, x_{n}, x_{1,2}, x_{2,3}, \ldots, x_{n-1, n}, \ldots, x_{1,2}, \ldots, n$ are real coefficients. For instance, setting $n=2$ leads to $\mathbb{C}^{2}=x_{0}+x_{1} i_{1}+x_{2} i_{2}+$ $x_{1,2} i_{1} i_{2}$. A $\mathbb{C}^{n}$ number has $2^{n} x$-coefficients: one $x_{0}$ for the real part, $n$ coefficients $x_{1}, x_{2}, \ldots, x_{n}$ for a single imaginary direction. All the other coefficients are for mixed imaginary directions with multiple $i_{j}$. Unlike quaternion [Shoemake 1985], the product between different imaginary units is commutative such that $i_{j} \cdot i_{k}=i_{k} \cdot i_{j}$ for $j \neq k$.

${ }^{1}$ The fact is Fourier differentiation still has the subtractive cancellation issue. Explicitly avoiding the subtraction is not a real cure of cancellation. This is out of scope of this paper, but numerical experiments clearly verify this.
Following the formulation in [Lantoine et al. 2012], the Taylor series expansion of $f^{\star}$ under a multicomplex perturbation is:

$$
\begin{aligned}
& f^{\star}\left(x_{0}+h i_{1}+\cdots+h i_{n}\right)=f^{\star}\left(x_{0}\right)+f^{\star(1)}\left(x_{0}\right) \cdot h \sum_{j=1}^{n} i_{j} \\
& +\frac{f^{\star(2)}\left(x_{0}\right)}{2} \cdot h^{2}\left(\sum_{j=1}^{n} i_{j}\right)^{2}+\cdots+\frac{f^{\star(n)}}{n !} \cdot h^{n}\left(\sum_{j=1}^{n} i_{j}\right)^{n}+\cdots .
\end{aligned}
$$

Here, $f^{\star(n)}$ is the $n$-th-order derivative of $f^{\star} .\left(\sum i_{j}\right)^{k}$ can be expanded following the multinomial theorem, and it contains products of mixed $k$ imaginary directions for the $k$-th-order term. We refer the reader to [Lantoine et al. 2012; Nasir 2013] for a detailed stepby-step derivation. Because $\left(\sum i_{j}\right)^{k} \neq\left(\sum i_{j}\right)^{l}$ for $k \neq l$, Eq. (24) allows us to approximate an arbitrary-order derivative by directly extracting the corresponding imaginary combination, just as we did in CSFD. In order to do so, $\operatorname{Im}(\cdot)$ should also be generalized to $\operatorname{Im}_{\kappa}(\cdot)$ to handle multiple imaginary directions:

$$
\operatorname{Im}_{\kappa}(z)=x_{\kappa} \in \mathbb{R}
$$

which picks a coefficient $x_{\kappa}$ that matches the imaginary combination of $\kappa$ (i.e. the subscripts combination of $i_{j}$ ).

The MCSFD approximation of the $n$-th-order derivative can then be concisely formulated as:

$$
f^{(n)}\left(x_{0}\right)=\frac{\operatorname{Im}^{(n)}\left(f^{\star}\left(x_{0}+h i_{1}+h i_{2}+\ldots+h i_{n}\right)\right)}{h^{n}}+\mathrm{O}\left(h^{2}\right) .
$$

Similarly, $n$-th-order partial derivative can be approximated as:

$$
\frac{\partial^{n} f\left(x_{1}, \cdots, x_{p}\right)}{\partial x_{1}^{k_{1}} \cdots \partial x_{k}^{k_{p}}} \approx \frac{\operatorname{Im}^{(n)}\left(f^{\star}\left(x_{1}+h \sum_{j \in \Pi_{1}} i_{j}, \cdots, x_{p}+h \sum_{j \in \Pi_{p} i_{j}}\right)\right)}{h^{n}},
$$

where $\operatorname{Im}^{(n)}=\operatorname{Im}_{1,2, \ldots, n}$ is a shortcut notation, which picks the coefficient of the mixed imaginary direction of $i_{1} i_{2} \cdots i_{n} . \Pi_{j}=$ $\left\{\sum_{l=1}^{j-1} k_{l}+1, \cdots, \sum_{l=1}^{j} k_{l}\right\}$. By setting $n=2$ in Eqs. (26) and (27), elements of the Hessian matrix (of a function $f(x, y): \mathbb{R}^{2} \rightarrow \mathbb{R}$ ) can be easily obtained as:

$$
\left\{\begin{array}{l}
\frac{\partial^{2} f(x, y)}{\partial x^{2}} \approx \frac{\operatorname{Im}^{(2)}\left(f\left(x+h i_{1}+h i_{2}, y\right)\right)}{h^{2}} \\
\frac{\partial^{2} f(x, y)}{\partial y^{2}} \approx \frac{\operatorname{Im}^{(2)}\left(f\left(x, y+h i_{1}+h i_{2}\right)\right)}{h^{2}} \\
\frac{\partial^{2} f(x, y)}{\partial x \partial y}=\frac{\partial^{2} f(x, y)}{\partial y \partial x} \approx \frac{\operatorname{Im}^{(2)}\left(f\left(x+h i_{1}, y+h i_{2}\right)\right)}{h^{2}} .
\end{array}\right.
$$

The most pleasing advantage of MCSFD to us is its handy implementation. As long as CSFD is implemented, all the routines for CSFD can be recursively used for MCSFD. More importantly, all the acceleration techniques discussed in Sec. 5 are also inherited with MCSFD. The numerical performance of MCSFD is excellent as reported in Fig. 5, where we evaluate the second-order derivative of $f(x)=e^{x} /\left(x^{4}+x^{2}+1\right)$ at $x=4$, the same example used in Fig. 2. The actual derivative $f^{\prime \prime}(x)=\left(x^{8}-8 x^{7}+22 x^{6}-12 x^{5}+\right.$ 


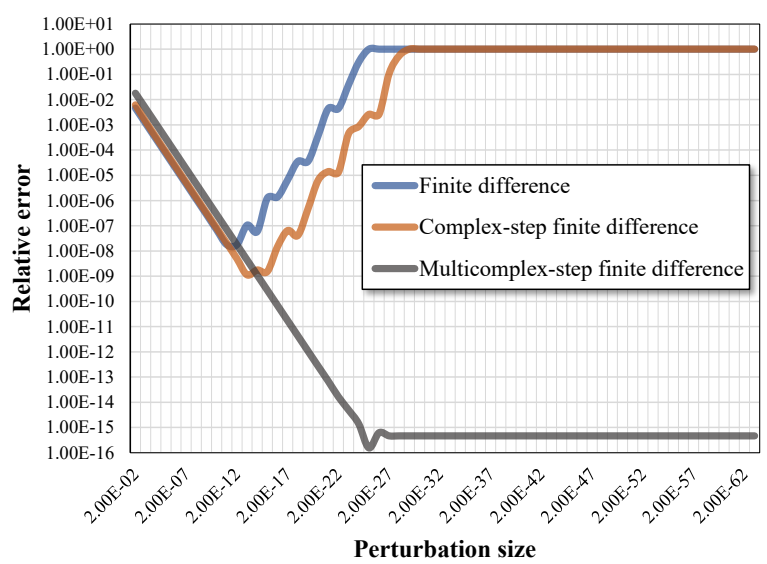

Fig. 5. The performance of MCSFD approximation. We use the same test function of $f(x)=e^{x} /\left(x^{4}+x^{2}+1\right)$ as in Fig. 2 and calculate its second-order derivative at $x=4$. The relative error w.r.t to value of analytic derivative is plotted against the size of the perturbation, ranging from $2^{-2}$ to $2^{-63}$.

$\left.21 x^{4}-12 x^{3}-4 x^{2}-4 x-1\right) e^{x} /\left(x^{4}+x^{2}+1\right)^{3}$ is used as the reference. In this example, second-order finite difference (Eq. (18)) has a similar behavior of its first-order counterpart. After $h$ hits a certain threshold $\left(\sim 1.0 \times 10^{-13}\right)$, the subtractive cancellation makes the numerator a numerical zero leading to a $100 \%$ relative error. The second-order CSFD approximation of Eq. (19) also suffers from this issue. MCSFD however, accurately approximates the second-order derivative. With a sufficiently small $h$, the relative error becomes comparable to the machine epsilon, and the approximation can be used to fully replace the analytic derivative.

\section{TENSOR FUNCTION}

Most examples we have discussed so far are real functions taking a single real-valued input. In many simulation problems, however, we deal with functions with a tensor input. If we know how each component of the input tensor contributes to the output, we can simply overload the corresponding calculation to evaluate the promoted function value. For instance, $f(\mathrm{X}): \mathbb{R}^{N \times N} \rightarrow \mathbb{R}=|\mathrm{X}|_{F}$ returns the Frobenius norm of the input matrix $X$. As we know the exact form of this function is: $f(\mathrm{X})=\sqrt{\sum \sum X_{i, j}^{2}}$, evaluating the partial derivative of $\partial f(\mathrm{X}) / \partial X_{i, j}$ is nothing more than fixing unrelated tensor components to pose $f$ as a scalar-input function.

However, there are also many functions that do not rely on an explicit formulation such as the one solving an input linear system:

$$
f(\mathrm{X}): \mathbb{R}^{N \times N} \rightarrow \mathbb{R}^{N}=\mathrm{X}^{-1} \text { a. }
$$

The exact inverse of a high-dimension matrix $\mathrm{X}$ is seldom given analytically. Instead, appropriate numerical routines like LU decomposition and forward-backward substitution are used to retrieve the function output. It is difficult for us to apply CSFD or MCSFD promotions without altering the underlying implementation of those numerical procedures.

An important advantage of CSFD/MCSFD is that one can exploit the Cauchy-Riemann (CR) formulation [Ahlfors 1973] to achieve (multi-)complex perturbations without overloading the complex arithmetic. CR form represents a multicomplex number in the form of a real matrix. Suppose $z^{1}=z_{0}^{0}+z_{1}^{0} i$, its CR form is a $2 \times 2$ matrix: $z^{1}=z_{0}^{0}+z_{1}^{0} i=\left[\begin{array}{cc}z_{0}^{0} & -z_{1}^{0} \\ z_{1}^{0} & z_{0}^{0}\end{array}\right]$, where $z^{1} \in \mathbb{C}^{1}$ and $z_{0}^{0}, z_{1}^{0} \in \mathbb{C}^{0}=\mathbb{R}$. Here, we use the superscript $(\cdot)^{n}$ to denote the order of a multicomplex number. The CR matrix of $z^{n}$ can be constructed recursively using the CR matrices of $z_{0}^{n-1}$ and $z_{1}^{n-1}$ following the definition of the multicomplex number (Eq. (22)) as:

$$
z^{n}=z_{0}^{n-1}+z_{1}^{n-1} i_{n} \in \mathbb{C}^{n}=\left[\begin{array}{cc}
z_{0}^{n-1} & -z_{1}^{n-1} \\
z_{1}^{n-1} & z_{0}^{n-1}
\end{array}\right] .
$$

Each of the $2 \times 2$ blocks in Eq. (30) is a $(n-1)$-order multicomplex number, which can be further expanded with $(n-2)$-order multicomplex numbers and so on. Eventually, the CR form of $z^{n}$ becomes a $2^{n} \times 2^{n}$ real matrix.

$\mathrm{CR}$ form can also be generalized for tensors i.e. $z_{0}^{0}$ and $z_{1}^{0}$ can be real-valued tensor quantities. As a result, $f(\mathbf{X})$ of Eq. (29) can be promoted as:

$$
f^{*}\left(\mathbf{X}^{*}\right)=\left[\begin{array}{cc}
\operatorname{Re}\left(\mathbf{X}^{*}\right) & -\operatorname{Im}\left(\mathbf{X}^{*}\right) \\
\operatorname{Im}\left(\mathbf{X}^{*}\right) & \operatorname{Re}\left(\mathbf{X}^{*}\right)
\end{array}\right]^{-1}\left[\begin{array}{cc}
\operatorname{Re}\left(\mathbf{a}^{*}\right) & -\operatorname{Im}\left(\mathbf{a}^{*}\right) \\
\operatorname{Im}\left(\mathbf{a}^{*}\right) & \operatorname{Re}\left(\mathbf{a}^{*}\right)
\end{array}\right] .
$$

Because all the tensors are now real quantities, Eq. (31) can be evaluated without involving any complex number calculations. The resulting function value is also the $\mathrm{CR}$ form of $f^{*}\left(\mathrm{X}^{*}\right)$, and we can extract its imaginary values from off-diagonal blocks. Fig. 6 reports another numerical experiment of using CR form to calculate first-order and second-order derivative of the inverse of a $3 \times 3$ matrix: $f(\mathbf{X})=\mathbf{X}^{-1} \in \mathbb{R}^{3 \times 3}$ w.r.t $X_{2,2}$ (i.e. the element resides at the second row and second column of $\mathbf{X})$. In this example, we generate a random $3 \times 3$ non-singular matrix, and compute its inverse matrix analytically. The exact formulation of the first-order and secondorder derivative of matrix inverse is:

$$
\frac{\partial f}{\partial X_{2,2}}=-\mathbf{X}^{-1} \frac{\partial \mathbf{X}}{\partial X_{2,2}} \mathbf{X}^{-1}, \quad \frac{\partial^{2} f}{\partial X_{2,2}^{2}}=-2 \mathbf{X}^{-1} \frac{\partial \mathbf{X}}{\partial X_{2,2}} \mathbf{X}^{-1} \frac{\partial \mathbf{X}}{\partial X_{2,2}} \mathbf{X}^{-1}
$$

and it is used as the reference.

The relative error of the numerical derivative computed using $\mathrm{CR}$ form as well as using the finite difference is plotted. We can see from Fig. 5 that CR form based CSFD and MCSFD also have excellent accuracy, while the regular finite difference still suffers with the subtractive cancellation.

\section{EXPERIMENTAL RESULTS}

We implemented CSFD/MCSFD on a desktop computer with an Intel i $78700 \mathrm{~K} \mathrm{CPU}$ and $32 \mathrm{~GB}$ memory. Both regular complex arithmetic and the generalized multicomplex arithmetic were implemented using $\mathrm{C}++$ double precision (64 bit on a x64 computer). While we believe CSFD/MCSFD will be useful for many graphics problems, in this paper we demonstrate its applications in modeling and simulating elastic objects. Unless specified, we set $h$ as $1.0 \times 10^{-40}$ in our experiments. Our general observation is that one can fully rely on CSFD/MCSFD-based derivative without any accuracy concerns. Performance-wise, accelerated CSFD/MCSFD is almost as efficient as analytic derivatives. We also compared CSFD/MCSFD with some widely used AD packages. While both 


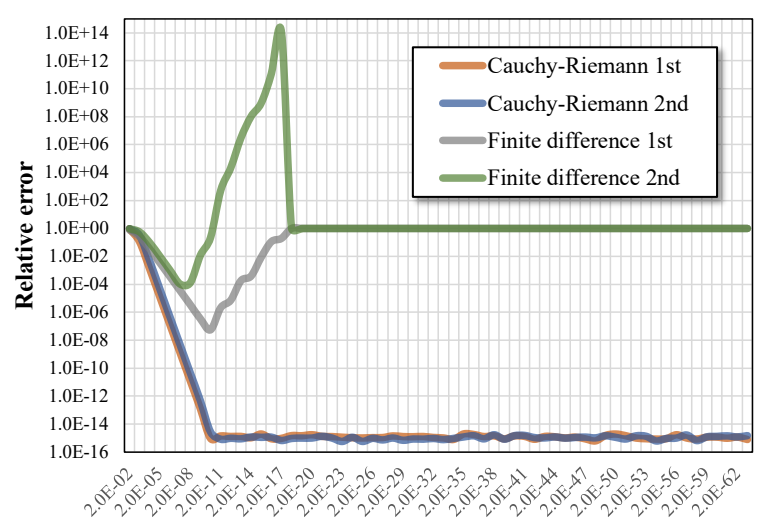

Perturbation size

Fig. 6. Cauchy-Riemann formula allows us to use existing linear algebra libraries to compute high-order numerical derivative without referring to an explicit complex promotion. In this example, we compute the first- and second-order derivative of $3 \times 3$ matrix inverse. CR-form based CSFD and MCSFD still have excellent accuracy compared with regular finite difference.

CSFD/MCSFD and AD produce accurate results in well-conditioned problems, CSFD/MCSFD excels at its robustness for nonsmooth functions, high-order generalization, and tensor extension. Accelerated CSFD/MCSFD is also much faster: it is over $20 \times$ faster than $\mathrm{C}++$ based $\mathrm{AD}$ packages and $\sim 300 \times$ faster than Python based $\mathrm{AD}$ packages.

Table 2. Time statistics of computing first- (1st), second- (2nd), and thirdorder (3rd) derivatives for 1 million times of the function: $f(x)=e^{x} /\left(x^{4}+\right.$ $\left.x^{2}+1\right)$ using CSFD/MCSFD and some popular AD packages.

\begin{tabular}{|c|c|c|c|c|c|}
\hline & CSFD & Adept (s) & CppAD (s) & ADOL-C (s) & $\operatorname{ad}(s)$ \\
\hline $1^{\text {st }}$ & $114 \mathrm{~ms}$ & $11.1(97 \times)$ & $8.2(72 \times)$ & $1.4(13 \times)$ & $72.1(632 \times)$ \\
\hline $2^{\text {nd }}$ & $242 \mathrm{~ms}$ & NA & $11.2(49 \times)$ & $5.9(24 \times)$ & $80.3(332 \times)$ \\
\hline $3^{\mathrm{rd}}$ & $768 \mathrm{~ms}$ & NA & NA & $51(62 \times)$ & NA \\
\hline
\end{tabular}

Comparison with $A D$ packages. In the first experiment, we would like to examine the efficiency of our accelerated CSFD/MCSFD as well as some widely used AD packages including Adept [Hogan 2014], CppAD [Bell 2012], ADOL-C [Griewank et al. 1996], and ad [Lee 2013]. The first three libraries are in $\mathrm{C}++$, and ad is a famous Python package. We record the time performance for evaluating derivatives (for 1 million times) of the function: $f(x)=e^{x} /\left(x^{4}+x^{2}+1\right)$ at $x=4$ (i.e. the one used in Figs. 2 and 5). The computation time for the analytic first- and second-order derivatives is $104 \mathrm{~ms}$ and $238 \mathrm{~ms}$ respectively, which is quite close to our CSFD/MCSFD taking $114 \mathrm{~ms}$ and $242 \mathrm{~ms}$. This function is smooth and differentiable, and all $\mathrm{AD}$ packages return accurate first-order derivative results successfully. Yet, our method is massively faster than AD packages as reported in Tab. 2. In general, the accelerated CSFD/MCSFD is dozens times faster than $\mathrm{C}++$ based $\mathrm{AD}$ packages and hundreds times faster than Python based ones. In this experiment, Adept does not support second-order derivative natively. CPpAD and ad only support high-order derivative up to the second order. $A D O L-C$ is the
Table 3. Computing the internal force and tangent stiffness matrix for $10 k$ linear tetrahedral elements of StVK and Neo-Hookean materials. Similar to Tab. 2, our accelerated CSFD/MCSFD is much faster than AD packages.

\begin{tabular}{c|c|c|c|c|c}
\hline & CSFD & Adept $(s)$ & CppAD $(s)$ & ADOL-C $(s)$ & ad $(s)$ \\
\hline StVK $1^{\text {st }}$ & $9 m s$ & $1.1(122 \times)$ & $0.8(90 \times)$ & $0.7(78 \times)$ & $7.1(786 \times)$ \\
${\text { StVK } 2^{\text {nd }}}$ & $101 \mathrm{~ms}$ & NA & $5.4(54 \times)$ & $5.2(52 \times)$ & $29(288 \times)$ \\
NH $1^{\text {st }}$ & $12 m s$ & $1.2(99 \times)$ & $0.8(66 \times)$ & $0.8(65 \times)$ & $7.2(580 \times)$ \\
NH $2^{\text {nd }}$ & $117 m s$ & NA & $5.6(48 \times)$ & $5.7(49 \times)$ & $31(268 \times)$ \\
\hline
\end{tabular}

most sophisticated package, which has dedicated sub-routines for second-order and high-order derivatives. Nevertheless, it is still more than one order slower than our method. ADOL-C becomes even slower for higher-order derivatives as it calls the first-order routine repeatedly for high-order cases (i.e. with its forward() routune). Python package is the slowest.

We also assess the robustness of $\mathrm{AD}$ packages for nonsmooth functions. Consider $f(x)=\log ^{2}\left(1-\sqrt{(x-1)^{2}}\right)$. Its analytic firstand second-order derivative can be easily derived as:

$$
f^{\prime}(x)=-\frac{2(x-1) \log \left(1-\sqrt{(x-1)^{2}}\right)}{\left(1-\sqrt{(x-1)^{2}}\right) \sqrt{(x-1)^{2}}},
$$

and

$$
f^{\prime \prime}(x)=-\frac{2\left(\log \left(1-\sqrt{(x-1)^{2}}\right)-1\right)}{\left(1-\sqrt{(x-1)^{2}}\right)^{2}} .
$$

We notice that $x=1$ is actually a singular point of the function. Without explicitly cancelling out $\sqrt{(x-1)^{2}}$ from Eqs. (32) and (33), AD packages that overload elementary arithmetic with differentiation rules tend to yield the division-by-zero error ${ }^{2}$. In this experiment, only Adept successfully returns the first-order derivative of this function, but It yields a \#IND error for the second-order case. All other AD packages return either NaN, \#IND, or ZeroDivisionError error. On the other hand, CSFD/MCSFD robustly handle this function derivative without any special treatments.

We observe similar results when applying CSFD/MCSFD and AD in deformable simulation computations. Tab. 3 lists the time performance of computing the internal force and tangent stiffness matrix for $10 k$ linear tetrahedral elements of StVK and Neo-Hookean materials, which are the first- and second-order partial derivatives of the energy function. The analytic formulations of those two energies are known. We use Vega library [Barbič et al. 2012] to compute the analytic force and stiffness matrix. For the StVK material, it takes $11.8 \mathrm{~ms}$ and $103.5 \mathrm{~ms}$ for the first- and second-order derivatives. For the Neo-Hookean material, the computation time is $12.1 \mathrm{~ms}$ and $112.5 \mathrm{~ms}$ respectively. This performance measure is close to our accelerated CSFD and MCSFD as shown in the table. In this experiment, most $\mathrm{AD}$ packages deliver correct results (expect for Adept) but they are all much slower than accelerated CSFD and MCSFD. It is also common, in practice, to resort to symbolic differentiation tools like Mathematica or Maple. For instance, Maple

\footnotetext{
${ }^{2}$ We may be able to avoid this numerical instability of $\mathrm{AD}$ by expanding and simplifying the derivative function. But if we choose to do so, we are literally deriving the analytic formula of the derivative function, and why do we bother to use AD?
} 

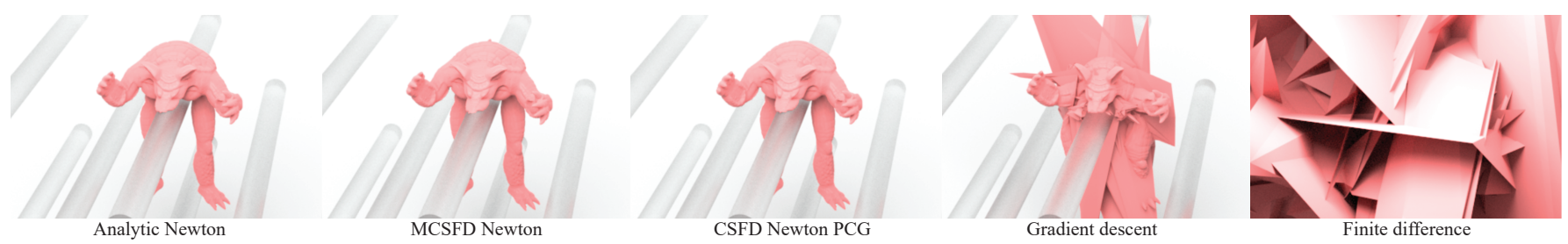

Fig. 7. The Armadillo model falls quickly and hits a glassy rod. Due to the sharp collision, gradient descent method [Wang and Yang 2016] yields artifact because the residual is not sufficiently reduced. Regular finite difference method crashes instantly. Newton's method with MCSFD-based Hessian yields the same result as using the analytic Newton. Newton-PCG with CSFD-based directional derivative also has the same result.
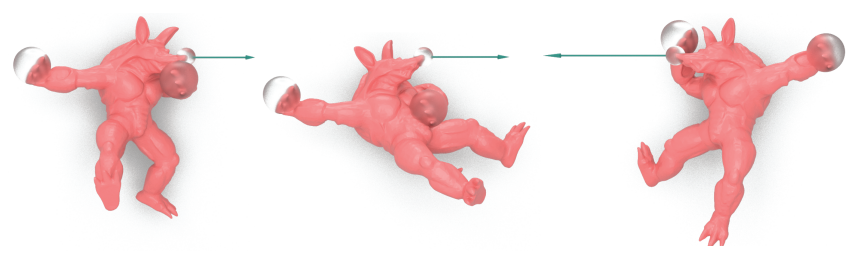

Fig. 8. We simulate a Neo-Hookean Armadillo model using Newton's method. The Armadillo has 69,074 elements. The gradient and Hessian of the target function $f$ (i.e. Eq. (35)) is approximated using numerical CSFD/MCSFD. The result is identical to the one computed using analytic gradient and Hessian.

package takes $\sim 1.5 s$ to yield the symbolic formulation of the firstorder energy gradient for the StVK model, which consists of over 800 terms. Clearly, without further simplifications, directly importing them to the simulator is redundant and inefficient.

\subsection{Application I: Accurate Nonlinear Optimization}

Dynamic simulation of a deformable object requires solving a nonlinear system of the force equilibrium. For instance, the implicit Euler time integration scheme leads to:

$$
\mathbf{M}\left(\mathbf{u}_{n+1}-\mathbf{u}_{n}-\Delta t \dot{\mathbf{u}}_{n}\right)=\Delta t^{2}\left(\mathbf{f}_{i n t}\left(\mathbf{u}_{n+1}\right)+\mathbf{f}_{\text {ext }}\right),
$$

where $\mathbf{M}$ is the mass matrix. $\mathbf{f}_{\text {int }}$ and $\mathbf{f}_{\text {ext }}$ stand for the elastic internal force and the external force. The subscript $(\cdot)_{n}$ denotes the time integration step, and $\Delta t$ is the time step size. $\mathbf{u}_{n+1}$ is the unknown displacement vector we want to compute. This equilibrium is often treated as an optimization problem known as its variational form [Liu et al. 2013; Stern and Desbrun 2006] of:

$$
\arg \min _{\mathbf{u}} f(\mathbf{u}), \quad f(\mathbf{u})=\frac{1}{\Delta t^{2}}\left\|\mathbf{M}^{\frac{1}{2}}\left(\mathbf{u}-\mathbf{u}^{*}\right)\right\|^{2}+E(\mathbf{u}),
$$

where $\mathbf{u}^{*}=\mathbf{u}_{n}+\Delta t \dot{\mathbf{u}}_{n}+h^{2} \mathbf{M}^{-1} \mathbf{f}_{\text {ext }}$ is a known vector. $E$ is the nonlinear elastic energy. Eq. (35) can be solved using the classic Newton's method, which approximates $f(\mathbf{u})$ with a quadratic form and calculates an incremental improvement of $\Delta \mathbf{u}$ as $\Delta \mathbf{u}=-\mathbf{H} \cdot \partial f / \partial \mathbf{u}$. Matrix $\mathbf{H}$ is the Hessian matrix, and it is the second-order partial derivative of $f: \mathbf{H}=\partial^{2} f / \partial \mathbf{u}^{2}$. We simulate nonlinear dynamics of a Neo-Hookean Armadillo (with 69, 074 elements) using Newton's method and drag its mouth back and forth. The gradient and Hessian of $f$ are approximated with CSFD/MCSFD. The elastic energy density $E$ of the Neo-Hookean material is

$$
E_{N H}=\lambda(J-1)^{2}+\mu\left(J^{-2 / 3} I_{1}-3\right)
$$

where $J=|\mathbf{F}|$ is the determinant of the deformation gradient $\mathbf{F}$, and $I_{1}=\operatorname{tr}\left(\mathbf{F}^{\top} \mathbf{F}\right) . \lambda$ and $\mu$ are Lamé constants. In our CSFD/MCSFD implementation, we treat $E$ as a nested composite function $E_{N H}=$ $E_{1}(J(\mathbf{F}))+E_{2}\left(I_{1}(\mathbf{F})\right)$. Snapshots of the deformed Armadillo are reported in Fig. 8. This animation is identical to the one obtained using analytic gradient and Hessian.

Alternatively, one may also use the Newton-PCG method, which replaces the direct solver used at each Newton iteration with an iterative PCG solver. As explained in [Yang et al. 2015], each NewtonPCG iteration calculates the product of $\left.K\right|_{\mathbf{u}_{0}} \cdot \mathbf{p}$, where $\left.K\right|_{\mathbf{u}_{0}}$ is the current tangent stiffness matrix at $\mathbf{u}=\mathbf{u}_{0}$, and $\mathbf{p}$ is a known displacement vector. This product can also be understood as the directional derivative of the energy function $E$ and be numerically computed via CSFD as:

$$
\left.\mathbf{K}\right|_{\mathbf{u}_{0}} \cdot \mathbf{p}=\left.\frac{\partial^{2} E}{\partial \mathbf{u}^{2}}\right|_{\mathbf{u}_{0}} \cdot \mathbf{p}=\left.\nabla_{\mathbf{p}} E\right|_{\mathbf{u}_{0}} \approx \frac{\operatorname{Im}\left(E^{*}\left(\mathbf{u}_{0}+h \cdot \mathbf{p} i\right)\right)}{h} .
$$

As shown in Fig. 7, CSFD-based directional derivative is also highly accurate, which produces the same result of analytic Newton and MCSFD Newton. The regular finite difference crashes immediately when the Armadillo collides with the glassy rod.

\subsection{Application II: Intuitive Hyperelastic Simulation}

For hyperelastic models, the form of the elastic energy (i.e. Eq. (35)) solely determines the deformed shape given inertial and external forces. Hyperelastic energy is typically defined based on three isotropic invariants of the deformation gradient: $I_{1}=\operatorname{tr}\left(\mathbf{F}^{\top} \mathbf{F}\right)$, $I_{2}=\operatorname{tr}\left(\left(\mathbf{F}^{\top} \mathbf{F}\right)^{2}\right)$, and $I_{3}=\left|\mathbf{F}^{\top} \mathbf{F}\right|^{2}$. Intuitively, $I_{1}$ measures the length change of the deformation; $I_{2}$ measures the area change of the deformation; and $I_{3}$ measures the volume change of the deformation. As long as the internal force $\partial E / \partial \mathbf{u}$ and the tangent stiffness matrix $\partial^{2} E / \partial \mathbf{u}^{2}$ are available, the dynamic behavior of the deformable

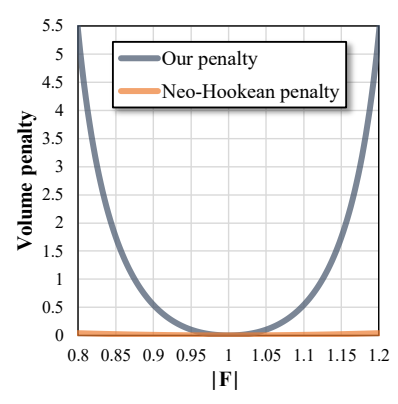

Fig. 10. We design a new volume penalty term of $\log ^{2}\left(1-4(J-1)^{2}\right), \quad$ which yields much bigger internal forces when $J=|\mathbf{F}|$ deviates from 1 than the regular Neo-Hookean volume penalty of $(J-1)^{2}$ does. body can be simulated using standard FEM. The closed-form formulation of $\partial E / \partial \mathbf{u}$ and $\partial^{2} E / \partial \mathbf{u}^{2}$ for some material models such as co-rotational model, StVK model, Neo-Hookean model are available 

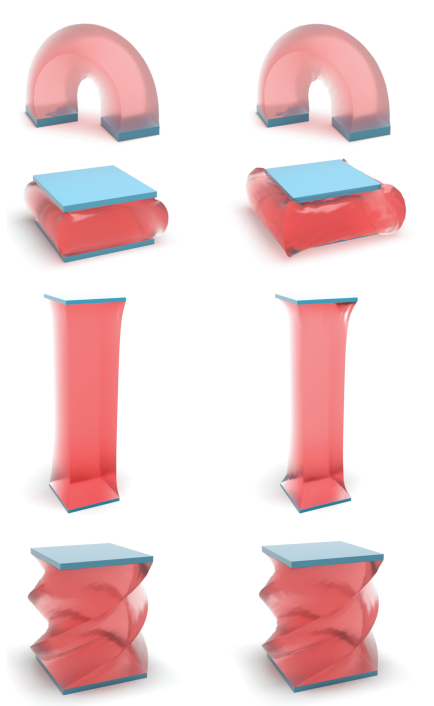

Arruda-Boyce
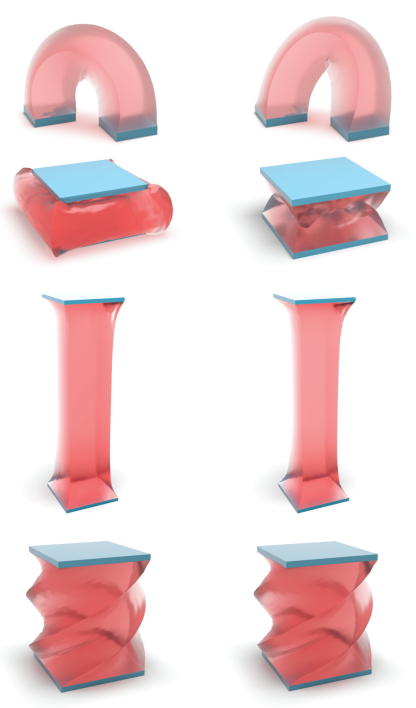

Fung
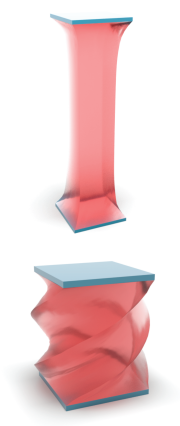

Mooney-Rivlin
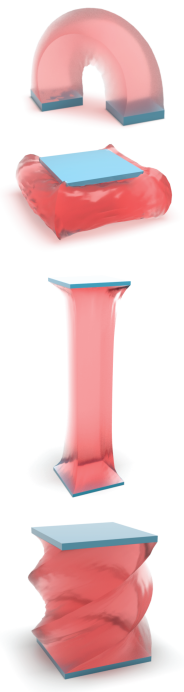

Neo-Hookean
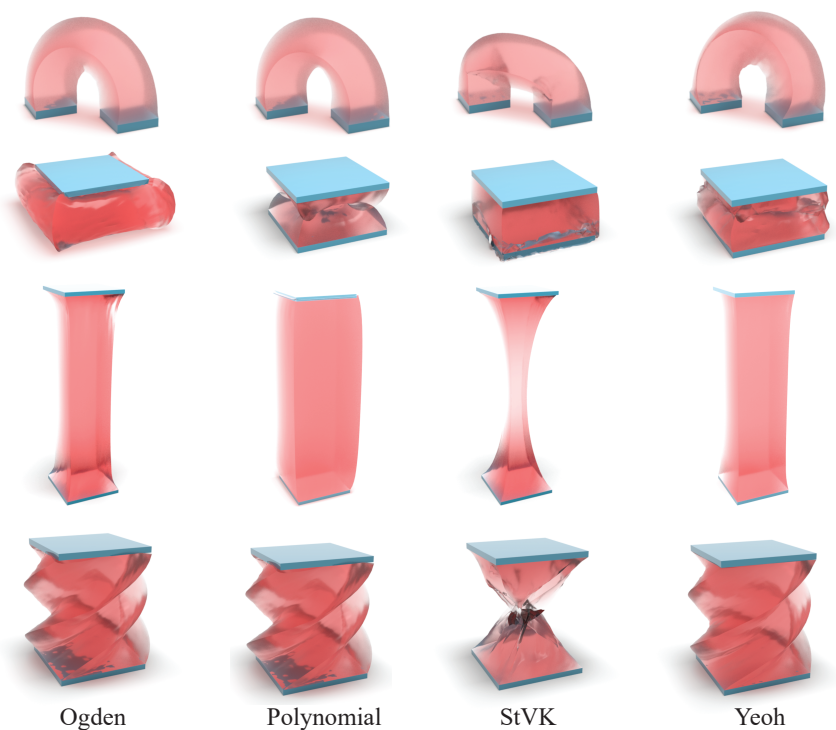

Fig. 9. CSFD/MCSFD allows the user to easily simulate all kinds of hyperelastic materials. The figure reports the material behaviors under standard bending, compressing, stretching, and twisting tests of a box model with 14, 678 elements. From left to right, each column gives the result of ArrudaâA̧̧̧oyce, Fung, Mooney-Rivlin, Neo-Hookean, Ogden, Polynomial, invertible StVK [Irving et al. 2004] (for improved stability), and Yeoh materials.

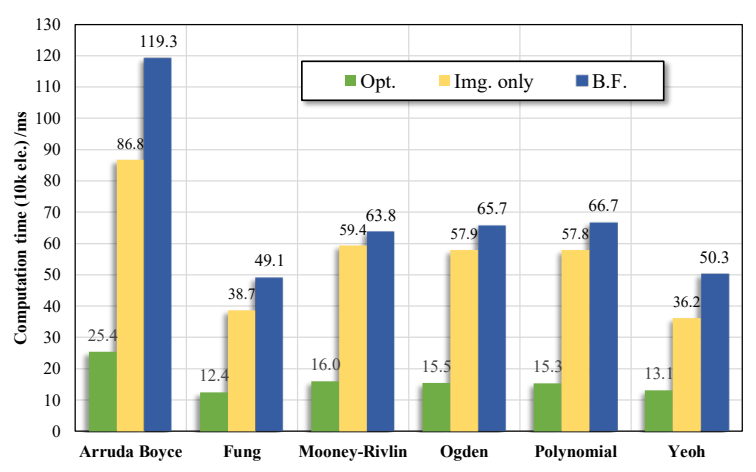

Fig. 11. Timing information of CSFD/MCSFD derivative in simulating various hyperelastic materials. Opt. is the optimized CSFD/MCSFD computation time. Img. only is the time without computing the real part of the promoted energy functions. B.F. is the computation time using a brute-force CSFD/MCSFD implementation.

in the literature [Bonet and Wood 1997; Sifakis and Barbic 2012; Smith et al. 2018]. However, there are many other materials such as Fung, Mooney-Rivlin, Ogden, Yeoh, Arruda Boyce models or the more general Polynomial model. Their energy structure can be easily followed, but deriving the actual formulation of force and stiffness matrix prevents these materials from being more widely employed by the graphics community. CSFD and MCSFD allow us to conveniently simulate hyperelastic materials with light-weight implementation efforts. As reported in Fig 9, we simulate all of those materials using CSFD/MCSFD under standard bending, compressing, stretching and twisting tests. In this experiment, we use

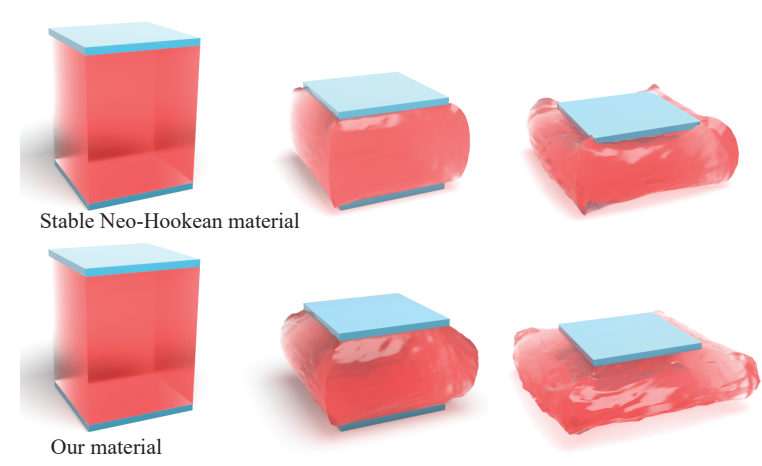

Fig. 12. Our new material (Eq. (38)) with a more aggressive volume penalty term is able to better preserve the volume of this jelly box during the compression than the stable Neo-Hookean material [Smith et al. 2018].

invertible StVK energy [Irving et al. 2004] to improve the stability of the regular StVK material. Timing information of different CSFD/MCSFD implementations is compared in Fig. 11.

In many situations, the user wants to use customized materials for specific needs in an animation scenario. For instance Smith and colleague [2018] proposed a new Neo-Hookean-like hyperelastic energy for a stable integration and volume preservation under large deformation. Using CSFD/MCSFD, users can freely explore various such energy densities without tedious derivations for internal force and Hessian. For instance, we design a new hyperelastic model:

$$
E_{\text {volume }}=\mu\left(J^{-2 / 3} I_{1}-3\right)+\frac{\lambda}{2} \log ^{2}\left(1-4(J-1)^{2}\right) .
$$

As plotted in Fig. 10, $E_{\text {volume }}$ triggers a much stronger resistance force to when $J=|\mathbf{F}|$ deviates from 1 and thus, better preserves the volume (i.e. see Fig. 12). In this example, the rest-shape volume 
of the jelly box is 0.64 . After compressing its height by $65 \%$, the new volume of the jelly box becomes 0.63 with $E_{\text {volume }}$ and 0.61 with the stable Neo-Hookean material [Smith et al. 2018]. While numbers look close, we can clearly see that the compressed box is much wider spread out with $E_{\text {volume }}$.

CSFD/MCSFD can deal with even more complicated energies. Another example is reported in Fig. 13. In this example, we use an examplebased hyperelastic energy as in [Martin et al. 2011], which has two target shapes, each of which embeds a smiling face ()) or a sad face $:-$ on the surface. We design this energy to be the function of the bending orientation so that corresponding internal forces arise when the box is bent to a cer-
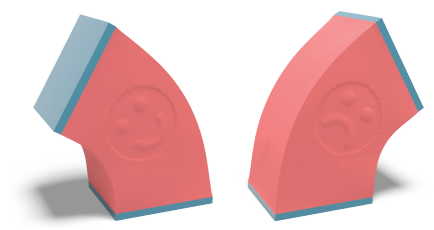

Fig. 13. Example-based hyperelastic energy can also be easily handled with CSFD/MCSFD. We make the energy a the function of bending angle so that a smiling face appears when the box bends to left and a sad face appears when the box bends to right. This box model has 14, 678 elements. tain direction. CSFD/MCSFD frees us from formulating the animation system and to quickly toy with many of such examples to achieve more interesting animations.
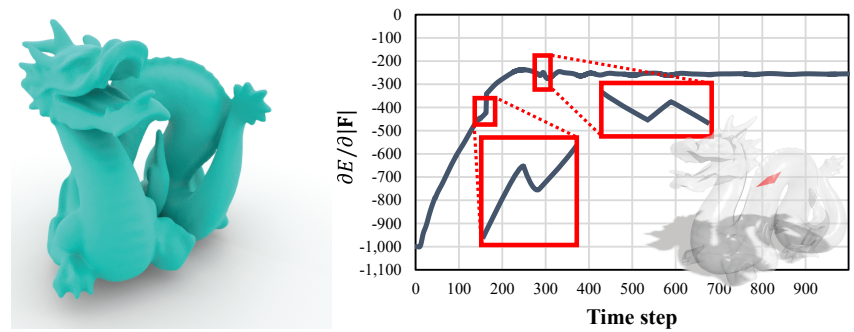

Fig. 14. Complicated energy formulation as Eq. (39) could hide singularities that are unfriendly for AD. CSFD/MCSFD can tackle this issue robustly.

For a customized material, it is possible that the user-specified energy has some singularities due to its complex formulation. In this case, $\mathrm{AD}$ packages, regardless of their slow performance, could even fail the simulation if any element reaches the singularity. To better elaborate this, we create another energy with the form of:

$$
E_{\text {singular }}=\mu\left(J^{-2 / 3} I_{1}-3\right)+\lambda(J-1)^{2}+\sqrt{\cos ^{2} 4(J-1)}-1 .
$$

As shown in Fig. 14, if we slowly bend the dragon with this material using $\mathrm{AD}$, the system crashes with the division-by-zero error when an element hits the singular point. CSFD/MCSFD is robust in such situations. Referring to Eq. (7), it is easy to see that as long as $f\left(x_{0}\right)$ exists, $f^{*}\left(x_{0}+h i\right)$ also exists because it is perturbed orthogonally towards the real domain and never touches the real-valued singularity. Therefore, CSFD always returns a well-estimated derivative value because $h$ is also nonzero.

\subsection{Application III: Expressive Model Reduction}

Model reduction is a widely-used technique to produce real-time deformable animation. This technique needs a pre-built subspace,

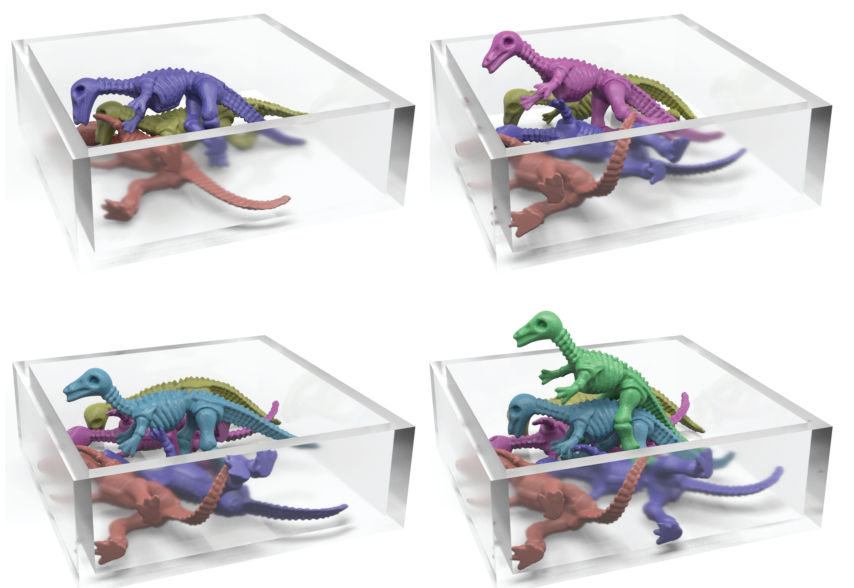

Fig. 15. Real-time simulation of six falling dinosaur models using modal derivative (30 modes for each dinosaur). The first-order derivative modes are computed using CSFD, and we use Fung, Mooney-Rivlin, Ogden, Yeoh, ArrudaâĂŞBoyce and Polynomial materials for each dinosaur.

which defines all the possible deformations of the deformable body. The standard method for subspace construction is based on the modal analysis [Pentland and Williams 1989], which provides the optimal vibrational modes around the rest shape. For nonlinear models, we need to compute derivative modes that first-order approximate a low-frequency nonlinear vibration [Barbič and James 2005; Yang et al. 2015]. Computing derivative modes requires the calculation of the force Hessian (i.e. the third-order derivative of $E$ ). Therefore, this powerful technique is normally used only for the StVK material, whose stiffness matrix is quadratic w.r.t to the displacement vector. Applying nonlinear model reduction to other materials using modal derivative is less exploited due to the barrier of computing high-order energy gradients. CSFD/MCSFD allows us to build expressive and compact subspace easily for any given hyperelastic material. Fig. 15 shows snapshots of a real-time simulation of six falling dinosaur models using 30 first-order modal derivatives. Each dinosaur model has 356, 48 elements, and they are of Fung, Mooney-Rivlin, Ogden, Yeoh, Arruda Boyce, and Polynomial materials. Yang and colleagues [2015] introduced a method that generalizes modal derivative to higher-order nonlinear shape approximation. This method can also be readily implemented with MCSFD. As shown in Fig. 16, we apply a circular force to bow the dinosaur model. Second-order modal derivatives are able to capture extreme bending effects. In this experiment, the hyperelastic material of Eq. (38) has a strong volume preserving term, which prevents this material from being extremely bent as other materials under the same external forces.

\subsection{Application IV: Convenient Inverse Design}

A lot of design problems tweak a collection of parameters to make sure that the simulated result matches certain specific measures like the maximum stress, deflection magnitude and so on. While there are many techniques (e.g. the well-known adjoint method) that are 


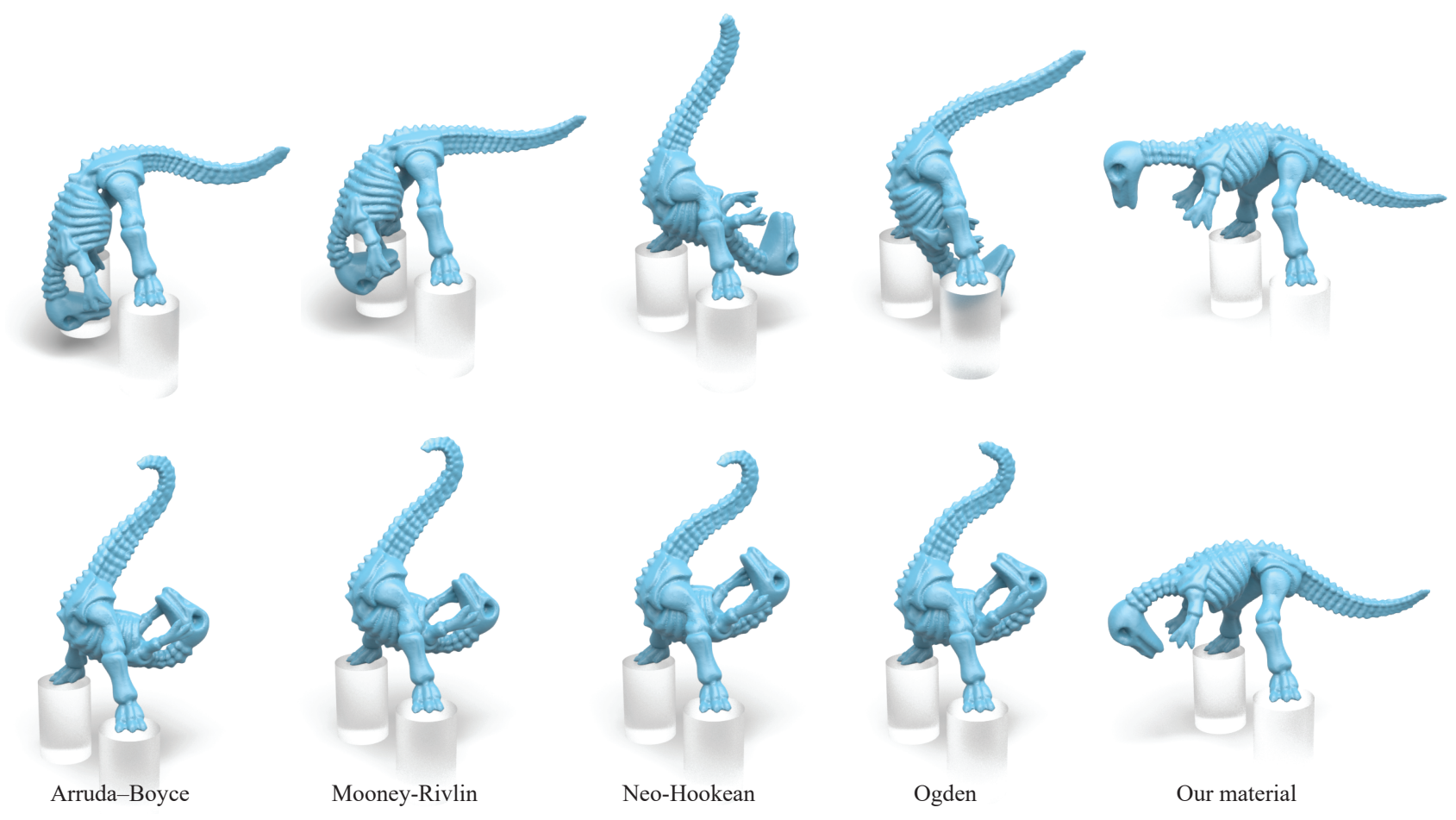

Fig. 16. MCSFD allows us to compute higher-order modal derivatives that capture extreme bending effects of the dinosaur model (using 30 second-order derivative modes). Interestingly, the hyperelastic energy of Eq. (38), because of its strong resistance to volume change, cannot be bent as hard as other materials under the same external force.
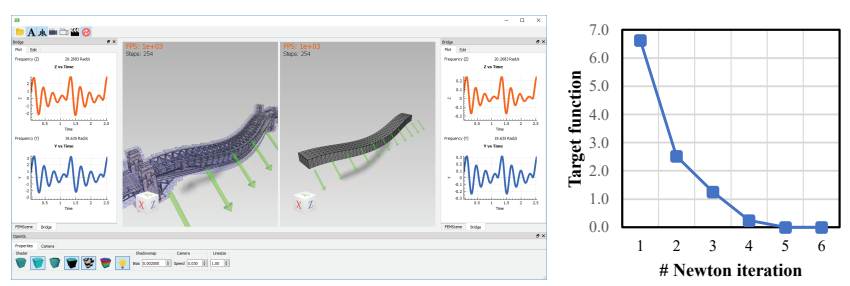

Fig. 17. We develop a system with an intuitive interface for the linear frequency design (left). The error reduces quickly along Newton iterations, with the Hessian accurately computed from MCSFD. (right)

capable of handling those problems, we show that CSFD/MCSFD is also a convenient alternative to deal with inverse simulations.

In Fig. 1, we show an example where the user wants to adjust the linear vibration frequencies of a bridge for a given external wind field by changing three primary geometry parameters: length $l$, width $w$ and the height of the arch top $t$. For an intuitive visualization of a frequency pattern, our system allows the user to apply this wind field to a standard rectangular beam (with two ends fixed) and to change its geometry/material to generate a preferred vibration pattern (see Fig. 17). The principle vibration of a linear structure under a given direction $\mathbf{u}$ is described by the Rayleigh quotient defined as $\omega^{2}=\mathbf{u}^{\top} \mathbf{K u} / \mathbf{u}^{\top} \mathbf{M u}$. The wind is modeled as an acceleration field a meaning $\mathbf{u}=\mathrm{K}^{-1} \mathrm{Ma}$. As a result, the frequency design procedure can be formulated as an optimization problem of:

$$
\arg \min _{l, w, t} f, \quad f(l, w, t)=\left\|\omega^{* 2}-\frac{\mathbf{a}^{\top} \mathbf{M} \mathbf{K}^{-1} \mathbf{M a}}{\mathbf{a}^{\top} \mathbf{M} \mathbf{K}^{-1} \mathbf{M K}^{-1} \mathbf{M a}}\right\|^{2},
$$

where $\omega^{* 2}$ is our target frequency. $\mathbf{M}=\mathbf{M}(l, w, t)$ and $\mathbf{K}=\mathbf{K}(l, w, t)$ are tensor functions of the unknown geometry parameters $l, w, t$ to be optimized. In this example, we use the CR form (Eq. (31)) to promote $\mathbf{M}(l, w, t)$ and $\mathbf{K}(l, w, t)$, and Newton's method is used to solve Eq. (40). Thanks to the accurate Hessian obtained by MCSFD, our solver quickly finds the optimal geometry only with few iterations.

\section{CONCLUSION AND FUTURE WORK}

In this paper, we show how to accelerate and generalize the complexstep finite difference to efficiently obtain an accurate numerical derivative of an arbitrary order. Its superior precision comes from complex or multicomplex promotion of the target function, which avoids the subtractive cancellation issue in standard finite difference methods. Without worrying about losing many significant digits during the calculation, CSFD and MCSFD allow us to have a very small perturbation to obtain a numerical derivative at the precision of machine epsilon implying it is as accurate as the analytic derivative. We propose a collection of acceleration techniques that avoid redundant and costly calculations induced by the complex promotion. Therefore our CSFD and MCSFD are as efficient as analytic derivative, but we are freed from the derivation for the actual differentiation. We show how this numerical algorithm can be applied 
for physics-based deformable simulation. Indeed, we believe that this method could be useful in a variety of graphics problems.

The limitation of this method may be it requires a dedicated implementation in order to achieve a good performance. When CR form is used, the computation quickly becomes prohibitive if one wants to evaluate higher-order derivatives for a tensor-valued function. However, if the efficiency is not the primary concern, one can implement CSFD and MCSFD quickly based on any existing complex arithmetic library. In the future, we would like to fully leverage this new method to attack other challenging computational problems. For instance, to perform the imaginary perturbation along the time domain to get a better time integration. It is also of great interests to us to apply this method to machine learning and other similar problems where optimizing complicated functions is required.

\section{ACKNOWLEDGMENTS}

We thank reviewers for their professional and constructive comments. Ran Luo and Yin Yang are partially supported by National Science Foundation (NSF) under grants No. 1717972 and No. 1845026 The views and conclusions contained herein are those of the authors and should not be interpreted as necessarily representing the official policies or endorsements, either expressed or implied, of NSF. Tianjia Shao is partially supported by NSF of China (No. 61772462, No. U1736217). Weiwei Xu is partially supported by NSF of China (No. 61732016), and the fundamental research fund for the central universities.

\section{REFERENCES}

Mark J Ablowitz and Athanassios S Fokas. 2003. Complex variables: introduction and applications. Cambridge University Press.

Rafael Abreu, Zeming Su, Jochen Kamm, and Jinghuai Gao. 2018. On the accuracy of the Complex-Step-Finite-Difference method. F. Comput. Appl. Math. 340 (2018), 390-403.

Lars V Ahlfors. 1973. Complex Analysis. 1979. (1973).

W Kyle Anderson, James C Newman, David L Whitfield, and Eric J Nielsen. 2001 Sensitivity analysis for Navier-Stokes equations on unstructured meshes using complex variables. AIAA journal 39, 1 (2001), 56-63.

RL Bagley. 2006. On Fourier differentiation - a numerical tool for implicit functions International fournal of Applied Mathematics 19, 3 (2006), 255.

David Baraff. 1989. Analytical methods for dynamic simulation of non-penetrating rigid bodies. In ACM SIGGRAPH Computer Graphics, Vol. 23. ACM, 223-232.

David Baraff. 1991. Coping with friction for non-penetrating rigid body simulation. ACM SIGGRAPH computer graphics 25, 4 (1991), 31-41.

David Baraff and Andrew Witkin. 1998. Large steps in cloth simulation. In Proceedings of the 25th annual conference on Computer graphics and interactive techniques. ACM, 43-54.

Jernej Barbič and Doug L James. 2005. Real-time subspace integration for St. VenantKirchhoff deformable models. In ACM transactions on graphics (TOG), Vol. 24. ACM, 982-990.

Jernej Barbič, Funshing Sin, and Eitan Grinspun. 2012. Interactive editing of deformable simulations. ACM Transactions on Graphics (TOG) 31, 4 (2012), 70.

Jernej Barbič, Fun Shing Sin, and Daniel Schroeder. 2012. Vega FEM Library. (2012).

Atilim Gunes Baydin and Barak A Pearlmutter. 2014. Automatic differentiation of algorithms for machine learning. arXiv preprint arXiv:1404.7456 (2014).

Bradley M Bell. 2012. CppAD: a package for $\mathrm{C}++$ algorithmic differentiation. Computational Infrastructure for Operations Research 57 (2012), 10.

Michael Betancourt. 2018. A Geometric Theory of Higher-Order Automatic Differentiation. arXiv preprint arXiv:1812.11592 (2018).

Javier Bonet and Richard D Wood. 1997. Nonlinear continuum mechanics for finite element analysis. Cambridge university press.

Patrick Brezillon, Jean-François Staub, Anne-Marie Perault-Staub, and Gérard Milhaud. 1981. Numerical estimation of the first order derivative: approximate evaluation of an optimal step. Computers \& Mathematics with Applications 7, 4 (1981), 333-347.

Robert Bridson. 2015. Fluid simulation for computer graphics. AK Peters/CRC Press.

$\mathrm{N}$ Butuk and J-P Pemba. 2003. computing CHEMKIN sensitivities using complex variables. Fournal of engineering for gas turbines and power 125, 3 (2003), 854-858.
Steve Capell, Seth Green, Brian Curless, Tom Duchamp, and Zoran Popović. 2002 Interactive skeleton-driven dynamic deformations. In ACM transactions on graphics (TOG), Vol. 21. ACM, 586-593.

Xiang Chen, Changxi Zheng, Weiwei Xu, and Kun Zhou. 2014. An asymptotic numerical method for inverse elastic shape design. ACM Transactions on Graphics (TOG) 33, 4 (2014), 95.

Jeffrey Fike and Juan Alonso. 2011. The development of hyper-dual numbers for exact second-derivative calculations. In 49th AIAA Aerospace Sciences Meeting including the New Horizons Forum and Aerospace Exposition. 886.

Jeffrey Alan Fike. 2013. Multi-objective optimization using hyper-dual numbers. Ph.D. Dissertation. Stanford university.

N Fleury, M Rausch Detraubenberg, and RM Yamaleev. 1993. Commutative extended complex numbers and connected trigonometry. Fournal of mathematical analysis and applications 180, 2 (1993), 431-457.

Rony Goldenthal, David Harmon, Raanan Fattal, Michel Bercovier, and Eitan Grinspun 2007. Efficient simulation of inextensible cloth. In ACM Transactions on Graphics (TOG), Vol. 26. ACM, 49 .

Andreas Griewank, David Juedes, and Jean Utke. 1996. Algorithm 755: ADOL-C: a package for the automatic differentiation of algorithms written in $\mathrm{C} / \mathrm{C}++. A C M$ Transactions on Mathematical Software (TOMS) 22, 2 (1996), 131-167.

Andreas Griewank and Andrea Walther. 2008. Evaluating derivatives: principles and techniques of algorithmic differentiation. Vol. 105. Siam.

Eitan Grinspun, Anil N Hirani, Mathieu Desbrun, and Peter Schröder. 2003. Discrete shells. In Proceedings of the 2003 ACM SIGGRAPH/Eurographics symposium on Computer animation. Eurographics Association, 62-67.

Gael Guennebaud, Benoit Jacob, et al. 2014. Eigen: a c++ linear algebra library. URL http://eigen. tuxfamily. org, Accessed 22 (2014).

Brian Guenter. 2007. Efficient symbolic differentiation for graphics applications. In ACM Transactions on Graphics (TOG), Vol. 26. ACM, 108.

Fabian Hahn, Sebastian Martin, Bernhard Thomaszewski, Robert Sumner, Stelian Coros, and Markus Gross. 2012. Rig-space physics. ACM transactions on graphics (TOG) 31, 4 (2012), 72.

Fabian Hahn, Bernhard Thomaszewski, Stelian Coros, Robert W Sumner, and Markus Gross. 2013. Efficient simulation of secondary motion in rig-space. In Proceedings of the 12th ACM SIGGRAPH/eurographics symposium on computer animation. ACM, $165-171$.

Robert Hecht-Nielsen. 1992. Theory of the backpropagation neural network. In Neural networks for perception. Elsevier, 65-93.

Robin J Hogan. 2014. Fast reverse-mode automatic differentiation using expression templates in C++. ACM Transactions on Mathematical Software (TOMS) 40, 4 (2014), 26.

IEEE. 1985. IEEE standard for binary floating-point arithmetic. Institute of Electrical and Electronic Engineers.

Geoffrey Irving, Joseph Teran, and Ronald Fedkiw. 2004. Invertible finite elements for robust simulation of large deformation. In Proceedings of the 2004 ACM SIGGRAPH/Eurographics symposium on Computer animation. Eurographics Association, $131-140$

Sanghaun Kim, Junghyun Ryu, and Maenghyo Cho. 2011. Numerically generated tangent stiffness matrices using the complex variable derivative method for nonlinear structural analysis. Computer Methods in Applied Mechanics and Engineering 200, 1-4 (2011), 403-413.

Yuki Koyama, Kenshi Takayama, Nobuyuki Umetani, and Takeo Igarashi. 2012. Real-time example-based elastic deformation. In Proceedings of the ACM SIGGRAPH/Eurographics Symposium on Computer Animation. Eurographics Association, $19-24$

Edna E Kramer. 1930. Polygenic functions of the dual variable $w=u+j v$. American fournal of Mathematics 52, 2 (1930), 370-376.

K-L Lai and JL Crassidis. 2008. Extensions of the first and second complex-step derivative approximations. f. Comput. Appl. Math. 219, 1 (2008), 276-293.

Gregory Lantoine, Ryan P Russell, and Thierry Dargent. 2012. Using multicomplex variables for automatic computation of high-order derivatives. ACM Transactions on Mathematical Software (TOMS) 38, 3 (2012), 16

Sonia Lebofsky. 2013. Numerically Generated Tangent Stiffness Matrices for Geometrically Non-Linear Structures. Ph.D. Dissertation.

Abraham Lee. 2013. ad: Fast, transparent first- and second-order automatic differentiation. (2013). http://pythonhosted.org/ad

Tiantian Liu, Adam W Bargteil, James F O’Brien, and Ladislav Kavan. 2013. Fast simulation of mass-spring systems. ACM Transactions on Graphics (TOG) 32, 6 (2013), 214.

Tiantian Liu, Sofien Bouaziz, and Ladislav Kavan. 2017. Quasi-newton methods for real-time simulation of hyperelastic materials. ACM Transactions on Graphics (TOG) 36, 4 (2017), 116a.

JN Lyness. 1968. Differentiation formulas for analytic functions. Math. Comp. (1968), 352-362.

James N Lyness. 1967. Numerical algorithms based on the theory of complex variable In Proceedings of the 1967 22nd national conference. ACM, 125-133. 
V Maple. 1994. Waterloo maple software. University of Waterloo, Version 5 (1994).

Charles C Margossian. 2018. A Review of automatic differentiation and its efficient implementation. Wiley Interdisciplinary Reviews: Data Mining and Knowledge Discovery (2018), e1305

Sebastian Martin, Bernhard Thomaszewski, Eitan Grinspun, and Markus Gross. 2011. Example-based elastic materials. In ACM Transactions on Graphics (TOG), Vol. 30 ACM, 72.

Joaquim RRA Martins, Peter Sturdza, and Juan J Alonso. 2003. The complex-step derivative approximation. ACM Transactions on Mathematical Software (TOMS) 29 3 (2003), 245-262.

Don Mitchell and Pat Hanrahan. 1992. Illumination from curved reflectors. In ACM SIGGRAPH Computer Graphics, Vol. 26. ACM, 283-291.

Arturo Montoya, Randal Fielder, Armando Gomez-Farias, and Harry Millwater. 2014 Finite-element sensitivity for plasticity using complex variable methods. Fournal of Engineering Mechanics 141, 2 (2014), 04014118.

HM Nasir. 2013. A new class of multicomplex algebra with applications. Mathematical Sciences International Research fournal 2, 2 (2013), 163-168.

Richard D Neidinger. 2010. Introduction to automatic differentiation and MATLAB object-oriented programming. SIAM review 52, 3 (2010), 545-563.

Jorge Nocedal and Stephen Wright. 2006. Numerical optimization. Springer Science \& Business Media.

Alex Pentland and John Williams. 1989. Good vibrations: Modal dynamics for graphics and animation. Vol. 23. ACM.

Agustí Pérez-Foguet, Antonio Rodríguez-Ferran, and Antonio Huerta. 2000. Numerical differentiation for local and global tangent operators in computational plasticity. Computer Methods in Applied Mechanics and Engineering 189, 1 (2000), 277-296.

Griffith Baley Price. 1991. An introduction to multicomplex spaces and functions. M Dekker.

Louis B Rall. 1981. Automatic differentiation: Techniques and applications. (1981).

Michael Renardy and Robert C Rogers. 2006. An introduction to partial differential equations. Vol. 13. Springer Science \& Business Media.

Boris Schäling. 2011. The boost $C++$ libraries. Boris Schäling.

Adriana Schulz, Jie Xu, Bo Zhu, Changxi Zheng, Eitan Grinspun, and Wojciech Matusik. 2017. Interactive design space exploration and optimization for cad models. $A C M$ Transactions on Graphics (TOG) 36, 4 (2017), 157.

Ken Shoemake. 1985. Animating rotation with quaternion curves. In ACM SIGGRAPH computer graphics, Vol. 19. ACM, 245-254.

Eftychios Sifakis and Jernej Barbic. 2012. FEM simulation of 3D deformable solids a practitioner's guide to theory, discretization and model reduction. In ACM SIGGRAPH 2012 Courses. ACM, 20.

Breannan Smith, Fernando De Goes, and Theodore Kim. 2018. Stable Neo-Hookean Flesh Simulation. ACM Transactions on Graphics (TOG) 37, 2 (2018), 12.

William Squire and George Trapp. 1998. Using complex variables to estimate derivatives of real functions. SIAM review 40, 1 (1998), 110-112.

Ari Stern and Mathieu Desbrun. 2006. Discrete geometric mechanics for variational time integrators. In ACM SIGGRAPH 2006 Courses. ACM, 75-80.

Demetri Terzopoulos, John Platt, Alan Barr, and Kurt Fleischer. 1987. Elastically deformable models. ACM Siggraph Computer Graphics 21, 4 (1987), 205-214.

Christoph W Ueberhuber. 2012. Numerical computation 1: methods, software, and analysis. Springer Science \& Business Media.

Andrew Voorhees, Harry Millwater, and Ronald Bagley. 2011. Complex variable methods for shape sensitivity of finite element models. Finite elements in analysis and design 47, 10 (2011), 1146-1156.

Huamin Wang and Yin Yang. 2016. Descent methods for elastic body simulation on the GPU. ACM Transactions on Graphics (TOG) 35, 6 (2016), 212.

Andrew Witkin. 1997. Physically Based Modeling: Principles and Practice Particle System Dynamics. SIGGRAPH Course notes (1997).

Stephen Wolfram et al. 1996. Mathematica. Cambridge university press Cambridge.

Hongyi Xu, Funshing Sin, Yufeng Zhu, and Jernej Barbič. 2015. Nonlinear material design using principal stretches. ACM Transactions on Graphics (TOG) 34, 4 (2015),

Guowei Yan, Wei Li, Ruigang Yang, and Huamin Wang. 2018. Inexact descent methods for elastic parameter optimization. In SIGGRAPH Asia 2018 Technical Papers. ACM 253.

Yin Yang, Dingzeyu Li, Weiwei Xu, Yuan Tian, and Changxi Zheng. 2015. Expediting precomputation for reduced deformable simulation. ACM Transactions on graphics (TOG) 34, 6 (2015)

\section{A ELEMENTARY COMPLEX PROMOTION}

The addition/subtraction and multiplication are trivial:

$$
\begin{aligned}
& f\left(x_{0}\right)=x_{0} \pm a \quad \rightarrow \quad f^{*}\left(x_{0}+h i\right)=x_{0} \pm a+h i \\
& f\left(x_{0}\right)=s \cdot x_{0} \quad \rightarrow \quad f^{*}\left(x_{0}+h i\right)=s x_{0}+s h i
\end{aligned}
$$

The division is treated as the multiplication of the conjugate:

$$
f\left(x_{0}\right)=\frac{a}{x} \rightarrow f^{*}\left(x_{0}+h i\right)=\frac{a}{r^{2}}\left(x_{0}-h i\right), r=\sqrt{x_{0}^{2}+h^{2}} .
$$

If the exponent of the power function $\left(x^{a}\right)$ is an integer i.e. $a=n \in \mathbb{Z}$, we can use the De Moivre's formula:

$$
f\left(x_{0}\right)=x^{n} \rightarrow f^{*}\left(x_{0}+h i\right)=r^{n}(\cos n \phi+\sin n \phi i),
$$

where $r \cos \phi=x_{0}$ and $r \sin \phi=h$ is the polar form of $x_{0}+h i$. On the other hand, $a=1 / m(m \in \mathbb{Z})$ makes $f\left(x_{0}\right)$ an $m$-root function, and the promotion is:

$$
f\left(x_{0}\right)=x_{0}^{\frac{1}{m}} \rightarrow f^{*}\left(x_{0}+h i\right)=r^{\frac{1}{m}}\left(\cos \frac{\phi+2 \pi k}{m}+\sin \frac{\phi+2 \pi k}{m} i\right) \text {. }
$$

Here, $k$ is an integer between 0 and $m-1$. In more general cases, when $a \in \mathbb{Q}$ is a rational number such that $a=n / m$, the power function of $x^{a}$ is split as $f(x)=y^{n}$ and $y=a^{1 / m}$.

The exponential function is promoted based on Euler's formula:

$$
f\left(x_{0}\right)=e^{x_{0}} \rightarrow f^{*}\left(x_{0}+h i\right)=e^{x_{0}}(\cos h+\sin h i) .
$$

The logarithmic promotion is the inverse of the exponential map, which can be obtained as:

$$
f\left(x_{0}\right)=\ln x_{0} \rightarrow f^{*}\left(x_{0}+h i\right)=\ln r+(\phi+2 \pi k) i, k \in \mathbb{Z} .
$$

Trigonometric functions can also be defined with complex numbers. According to Euler's formula, we have $\sin \alpha=\left(e^{\alpha i}-e^{-\alpha i}\right) / 2 i$. Substituting $\alpha$ with $x_{0}+h i$ leads to the promotion of $\sin x$ :

$f\left(x_{0}\right)=\sin x_{0} \rightarrow f^{*}\left(x_{0}+h i\right)=\frac{e^{h}+e^{-h}}{2} \sin x_{0}+\frac{e^{h}-e^{-h}}{2} \cos x_{0} i$.

Similarly, $\cos \alpha=\left(e^{\alpha i}+e^{-\alpha i}\right) / 2$ is promoted as:

$f\left(x_{0}\right)=\cos x_{0} \rightarrow f^{*}\left(x_{0}+h i\right)=\frac{e^{h}+e^{-h}}{2} \cos x_{0}-\frac{e^{h}-e^{-h}}{2} \sin x_{0} i$.

Note that the promotion of exponential and logarithmic functions of Eqs. (44) and (46) is not unique due to the periodicity. We can restrict the argument angle to $[0,2 \pi]$, that makes $k=0$. 